\title{
An explicit version of Faltings' Product Theorem and an improvement of Roth's lemma
}

\author{
by \\ JAN-HENDRIK EVERTSE (Leiden)
}

To Professor Wolfgang Schmidt on his 60th birthday

Introduction. In his paper Diophantine approximation on abelian varieties [1], Faltings proved, among other things, the following conjecture of Weil and Lang: if $A$ is an abelian variety over a number field $k$ and $X$ a subvariety of $A$ not containing a translate of a positive dimensional abelian subvariety of $A$, then $X$ contains only finitely many $k$-rational points. One of Faltings' basic tools was a new non-vanishing result of his, also proved in [1], the so-called (arithmetic version of the) Product Theorem. It has turned out that this Product Theorem has a much wider range of applicability in Diophantine approximation. For instance, recently Faltings and Wüstholz gave an entirely new proof [2] of Schmidt's Subspace Theorem [15] based on the Product Theorem.

Faltings' Product Theorem is not only very powerful for deriving new qualitative finiteness results in Diophantine approximation but, in an explicit form, it can be used also to derive significant improvements of existing quantitative results. In the present paper, we work out an explicit version of the arithmetic version of the Product Theorem; except for making explicit some of Faltings' arguments from [1] this did not involve anything new. By using the same techniques we improve Roth's lemma from [12]. Roth's lemma was used by Roth in his theorem on the approximation of algebraic numbers by rationals [12] and later by Schmidt in his proof of the Subspace Theorem [15].

In two subsequent papers we shall apply our improvement of Roth's lemma to derive significant improvements on existing explicit upper bounds for the number of subspaces in the Subspace Theorem, due to Schmidt [16] and Schlickewei [14] and for the number of solutions of norm form equations [17] and $S$-unit equations [13]. 
At the conference on Diophantine problems in Boulder in honour of W. M. Schmidt (26 June - 1 July, 1994), Wüstholz announced that his student R. Ferretti had independently obtained results similar to our Theorems 1 and 2. These results have been published in [3]. Part of the arguments used in the proof of Theorem 1 had already been worked out by van der Put [11] in his lecture at the conference "Diophantine approximation and Abelian varieties", Soesterberg, The Netherlands, 12-15 April 1992.

As the Product Theorem appears to have applications outside arithmetic algebraic geometry, we have tried to make this paper accessible to nongeometers with a modest knowledge of algebraic geometry.

1. Statement of the results. Let $\mathbf{n}=\left(n_{1}, \ldots, n_{m}\right)$ be a tuple of positive integers. For $h=1, \ldots, m$, denote by $\mathbf{X}_{h}$ the block of $n_{h}+1$ variables $X_{h 0}, \ldots, X_{h, n_{h}}$. For a ring $R$, denote by $R[\mathbf{X}]$ or $R\left[\mathbf{X}_{1}, \ldots, \mathbf{X}_{m}\right]$ the polynomial ring in the $\left(n_{1}+1\right)+\ldots+\left(n_{m}+1\right)$ variables $X_{h j}(h=1, \ldots, m, j=$ $\left.0, \ldots, n_{h}\right)$. For a tuple of non-negative integers $\mathbf{d}=\left(d_{1}, \ldots, d_{m}\right)$, denote by $\Gamma_{R}^{\mathbf{n}}(\mathbf{d})$ the $R$-module of polynomials in $R[\mathbf{X}]$ which are homogeneous of degree $d_{1}$ in the block $\mathbf{X}_{1}, \ldots$, homogeneous of degree $d_{m}$ in $\mathbf{X}_{m}$, i.e. the $R$-module generated by the monomials

$$
\mathbf{X}^{\mathbf{i}}:=\prod_{h=1}^{m} \prod_{j=0}^{n_{h}} X_{h j}^{i_{h j}} \quad \text { with } \sum_{j=0}^{n_{h}} i_{h j}=d_{h} \text { for } h=1, \ldots, m .
$$

Let $\Gamma_{R}^{\mathbf{n}}:=\bigcup_{\mathbf{d} \in\left(\mathbb{Z}_{>0}\right)^{m}} \Gamma_{R}^{\mathbf{n}}(\mathbf{d})$ be the set of polynomials which are homogeneous in each block $\mathbf{X}_{h}$ for $h=1, \ldots, m$. An $\mathbf{n}$-ideal of $R[\mathbf{X}]$ is an ideal generated by polynomials from $\Gamma_{R}^{\mathbf{n}}$. An essential $\mathbf{n}$-prime ideal of $R[\mathbf{X}]$ is an $\mathbf{n}$-ideal which is a prime ideal and which does not contain any of the ideals $\left(X_{h 0}, \ldots, X_{h, n_{h}}\right)(h=1, \ldots, m)$.

Let $k$ be an algebraically closed field and denote by $\mathbb{P}^{n}(k)$ the $n$-dimensional projective space over $k$. Every point $P \in \mathbb{P}^{n}(k)$ can be represented by a unique (up to a scalar multiple) non-zero vector $\mathbf{x}=\left(x_{0}, \ldots, x_{n}\right) \in k^{n+1}$ of homogeneous coordinates. Let again $\mathbf{n}=\left(n_{1}, \ldots, n_{m}\right)$ be a tuple of positive integers. Define the multi-projective space $\mathbb{P}^{\mathbf{n}}(k)$ as the cartesian product

$$
\mathbb{P}^{\mathbf{n}}(k):=\mathbb{P}^{n_{1}}(k) \times \ldots \times \mathbb{P}^{n_{m}}(k) .
$$

In what follows, $\mathbb{P}^{n}(k)$ with a non-bold face superscript denotes the $n$ dimensional (single-) projective space, and $\mathbb{P}^{\mathbf{n}}(k)$ with a bold-face superscript a multi-projective space. For $f \in \Gamma_{k}^{\mathbf{n}}$ and for $P=\left(P_{1}, \ldots, P_{m}\right) \in$ $\mathbb{P}^{\mathbf{n}}(k)$ with $P_{h} \in \mathbb{P}^{n_{h}}(k)$ for $h=1, \ldots, m$ we say that $f(P)=0($ or $\neq 0)$ if $f\left(\mathbf{x}_{1}, \ldots, \mathbf{x}_{m}\right)=0($ or $\neq 0)$ for any vectors of homogeneous coordinates $\mathbf{x}_{1}, \ldots, \mathbf{x}_{m}$, representing $P_{1}, \ldots, P_{m}$ respectively. This is well defined. A (Zariski-) closed subset of $\mathbb{P}^{\mathbf{n}}(k)$ is a set

$$
\left\{P \in \mathbb{P}^{\mathbf{n}}(k): f_{1}(P)=0, \ldots, f_{r}(P)=0\right\}
$$


(abbreviated $\left\{f_{1}=0, \ldots, f_{r}=0\right\}$ ), where $f_{1}, \ldots, f_{r} \in \Gamma_{k}^{\mathbf{n}} \backslash\{0\}$. A closed subset $X$ of $\mathbb{P}^{\mathbf{n}}(k)$ is called reducible if it is the union of two closed subsets $A, B$ of $\mathbb{P}^{\mathbf{n}}(k)$ with $A \subsetneq X, B \subsetneq X$, and irreducible otherwise. Every closed subset $X$ of $\mathbb{P}^{\mathbf{n}}(k)$ can be expressed uniquely as

$$
X=Z_{1} \cup \ldots \cup Z_{r},
$$

where $Z_{1}, \ldots, Z_{r}$ are irreducible closed subsets of $\mathbb{P}^{\mathbf{n}}(k)$ such that $Z_{i} \nsubseteq Z_{j}$ for $i, j \in\{1, \ldots, r\}, i \neq j$ (cf. [18], p. 23). $Z_{1}, \ldots, Z_{r}$ are called the irreducible components of $X$. We use the term "subvariety" exclusively for a projective subvariety, i.e. a closed irreducible subset.

There is a one-to-one correspondence between subvarieties of $\mathbb{P}^{\mathbf{n}}(k)$ and essential n-prime ideals $I$ of $k[\mathbf{X}]$ :

$$
I \leftrightarrow V(I)=\left\{P \in \mathbb{P}^{\mathbf{n}}(k): f(P)=0 \text { for all } f \in I\right\} .
$$

We say that the subvariety $V$ of $\mathbb{P}^{\mathbf{n}}(k)$ is defined over a subfield $k_{1}$ of $k$ if its corresponding prime ideal can be generated by polynomials with coefficients from $k_{1}$. An important class of subvarieties of $\mathbb{P}^{\mathbf{n}}(k)$ we will encounter are the product varieties

$$
Z_{1} \times \ldots \times Z_{m}=\left\{\left(P_{1}, \ldots, P_{m}\right): P_{h} \in Z_{h} \text { for } h=1, \ldots, m\right\},
$$

where $Z_{h}$ is a subvariety of $\mathbb{P}^{n_{h}}(k)$ for $h=1, \ldots, m$. It is a theorem (cf. [18], pp. 61-62) that the cartesian product of subvarieties of $\mathbb{P}^{n_{1}}(k), \ldots, \mathbb{P}^{n_{m}}(k)$, respectively, is a subvariety of $\mathbb{P}^{\mathbf{n}}(k)$.

Let $F \in \Gamma_{k}^{\mathbf{n}}$. For a tuple of non-negative integers $\mathbf{i}=\left(i_{h j}: h=\right.$ $\left.1, \ldots, m, j=0, \ldots, n_{h}\right)$ define the partial derivative of $F$ :

$$
F_{\mathbf{i}}:=\left(\prod_{h=0}^{m} \prod_{j=0}^{n_{h}} \frac{\partial^{i_{h j}}}{\partial X_{h j}^{i_{h j}}}\right) F .
$$

Let $\mathbf{d}=\left(d_{1}, \ldots, d_{m}\right)$ be a tuple of positive integers. For a tuple $\mathbf{i}$ as above, put

$$
(\mathbf{i} / \mathbf{d}):=\sum_{h=1}^{m} \frac{1}{d_{h}}\left(i_{h 0}+\ldots+i_{h, n_{h}}\right) .
$$

The index of $F$ with respect to $P \in \mathbb{P}^{\mathbf{n}}(k)$ and $\mathbf{d}$, notation $i_{\mathbf{d}}(F, P)$, is the largest number $\sigma$ such that

$$
F_{\mathbf{i}}(P)=0 \quad \text { for all } \mathbf{i} \text { with }(\mathbf{i} / \mathbf{d}) \leq \sigma .
$$

The index of $F$ at $P$ is some kind of weighted multiplicity of $F$ at $P$. The index is independent of the choice of homogeneous coordinates on $\mathbb{P}^{n_{h}}$ for $h=1, \ldots, m$. Namely, if for $h=1, \ldots, m, Y_{h 0}, \ldots, Y_{h, n_{h}}$ are linearly independent linear forms in $\mathbf{X}_{h}$, then the differential operators $\partial / \partial Y_{h j}$ are linear combinations of the $\partial / \partial X_{h j}$ and vice versa, hence the index does not change 
when in its definition the operators $\partial / \partial X_{h j}\left(j=0, \ldots, n_{h}\right)$ are replaced by $\partial / \partial Y_{h j}\left(j=0, \ldots, n_{h}\right)$ for $h=1, \ldots, m$.

For $\sigma \geq 0$, define the closed subset of $\mathbb{P}^{\mathbf{n}}(k)$,

$$
\begin{aligned}
Z_{\sigma} & =Z_{\sigma}(F, \mathbf{d}):=\left\{P \in \mathbb{P}^{\mathbf{n}}(k): i_{\mathbf{d}}(F, P) \geq \sigma\right\} \\
& =\left\{P \in \mathbb{P}^{\mathbf{n}}(k): F_{\mathbf{i}}(P)=0 \text { for all } \mathbf{i} \text { with }(\mathbf{i} / \mathbf{d}) \leq \sigma\right\} .
\end{aligned}
$$

$Z_{\sigma}$ need not be irreducible. The Product Theorem of Faltings [1], Thm. 3.1, states that if $Z$ is an irreducible component of $Z_{\sigma}$ and also of $Z_{\sigma+\varepsilon}$ for some $\varepsilon>0$, and if the quotients $d_{1} / d_{2}, \ldots, d_{m-1} / d_{m}$ are sufficiently large in terms of $\varepsilon$ and $m$, then $Z$ is a product variety. Below we have stated this result in an explicit form. The degree $\operatorname{deg} Z$ of a subvariety $Z$ of $\mathbb{P}^{n}$ is the number of points in the intersection of $Z$ with a generic linear projective subspace $L$ of $\mathbb{P}^{n}$ such that $\operatorname{dim} Z+\operatorname{dim} L=n$. The codimension of $Z$ is $n-\operatorname{dim} Z$.

TheOREM 1. Let $k$ be an algebraically closed field of characteristic 0 . Further, let $m$ be an integer $\geq 2, \mathbf{n}=\left(n_{1}, \ldots, n_{m}\right), \mathbf{d}=\left(d_{1}, \ldots, d_{m}\right)$ tuples of positive integers and $\sigma, \varepsilon$ reals such that $\sigma \geq 0,0<\varepsilon \leq 1$ and

$$
\frac{d_{h}}{d_{h+1}} \geq\left(\frac{m M}{\varepsilon}\right)^{M} \quad \text { for } h=1, \ldots, m-1,
$$

where $M:=n_{1}+\ldots+n_{m}$. Finally, let $F \in \Gamma_{k}^{\mathbf{n}}(\mathbf{d}) \backslash\{0\}$, and let $Z$ be an irreducible component of both $Z_{\sigma}(F, \mathbf{d})$ and $Z_{\sigma+\varepsilon}(F, \mathbf{d})$. Then $Z$ is a product variety

$$
Z=Z_{1} \times \ldots \times Z_{m}
$$

where $Z_{h}$ is a subvariety of $\mathbb{P}^{n_{h}}(k)$ for $h=1, \ldots, m$. Further, if $F$ has its coefficients in a subfield $k_{0}$ of $k$, then $Z_{1}, \ldots, Z_{m}$ are defined over an extension $k_{1}$ of $k_{0}$ with

$$
\left[k_{1}: k_{0}\right] \operatorname{deg} Z_{1} \ldots \operatorname{deg} Z_{m} \leq\left(\frac{m s}{\varepsilon}\right)^{s},
$$

where $s=\sum_{i=1}^{m} \operatorname{codim} Z_{i}$.

The idea behind the proof of Theorem 1 is roughly as follows. Any irreducible component $Z$ of both $Z_{\sigma}$ and $Z_{\sigma+\varepsilon}$ must have in some sense large multiplicity (analogously, if for a polynomial $f$ in one variable all derivatives of $f$ up to some order vanish at $P$ then $P$ has large multiplicity). On the other hand, using intersection theory one shows that the multiplicity of $Z_{\sigma}$ can be that large only if this component is a product variety.

Now let $k=\overline{\mathbb{Q}}$ be the field of algebraic numbers. We need estimates for the heights of $Z_{1}, \ldots, Z_{m}$ in terms of the height of $F$. First we define the height of $\mathbf{x}=\left(x_{0}, \ldots, x_{n}\right) \in \overline{\mathbb{Q}}^{n+1} \backslash\{\mathbf{0}\}$. Take any number field $K$ containing $x_{0}, \ldots, x_{n}$. Denote by $O_{K}$ the ring of integers of $K$ and let $\sigma_{1}, \ldots, \sigma_{f}, f=$ $[K: \mathbb{Q}]$, be the embeddings of $K$ into $\mathbb{C}$. Choose $\alpha \in O_{K} \backslash\{0\}$ such that 
$\alpha x_{0}, \ldots, \alpha x_{n} \in O_{K}$, let $\mathfrak{a}=\alpha x_{0} O_{K}+\ldots+\alpha x_{n} O_{K}$ be the ideal generated by $\alpha x_{0}, \ldots, \alpha x_{n}$, and $N \mathfrak{a}=\#\left(O_{K} / \mathfrak{a}\right)$ the norm of $\mathfrak{a}$. Then the height of $\mathbf{x}$ is defined by

$$
H(\mathbf{x}):=\left\{\frac{1}{N \mathfrak{a}} \prod_{j=1}^{f}\left(\sum_{i=0}^{n}\left|\sigma_{j}\left(\alpha x_{i}\right)\right|^{2}\right)^{1 / 2}\right\}^{1 / f} .
$$

It is easy to show that this does not depend on the choices of $\alpha$ and $K$. The height of a non-zero polynomial $F \in \overline{\mathbb{Q}}\left[X_{1}, \ldots, X_{n}\right]$ is defined by $H(F)=$ $H(\mathbf{x})$, where $\mathbf{x}$ is the vector of non-zero coefficients of $F$.

It is obvious that $H(\lambda \mathbf{x})=H(\mathbf{x})$ for every $\lambda \in \overline{\mathbb{Q}}^{*}$. Hence we can define a height on $\mathbb{P}^{n}(\overline{\mathbb{Q}})$ by $H(P)=H(\mathbf{x})$, where $\mathbf{x} \in \overline{\mathbb{Q}}^{n+1} \backslash\{\mathbf{0}\}$ is any vector representing $P$. By using the arithmetic intersection theory of Gillet and Soulé [5] for schemes over Spec $\mathbb{Z}$, Faltings defined a height $h(Z)$ for subvarieties $Z$ of $\mathbb{P}^{\mathbf{n}}(\overline{\mathbb{Q}})$ (cf. [1], pp. 552-553 and [7] for more details). This height is always $\geq 0$. Further, for points $P \in \mathbb{P}^{n}(\overline{\mathbb{Q}})$ we have

$$
h(P)=\log H(P) .
$$

Philippon [10] and Soulé [19] gave an explicit expression for the Faltings height of $Z$ in terms of the Chow form of $Z$. This is the unique (up to a constant) polynomial $F_{Z}$ in the $r+1$ blocks of $n+1$ variables $\zeta_{0}=$ $\left(\zeta_{00}, \ldots, \zeta_{0 n}\right), \ldots, \zeta_{r}=\left(\zeta_{r 0}, \ldots, \zeta_{r n}\right)$, where $r=\operatorname{dim} Z$ such that $F_{Z}$ has degree $\operatorname{deg} Z$ in each block $\zeta_{i}(i=0, \ldots, r)$ and such that $F_{Z}\left(\zeta_{0}, \ldots, \zeta_{r}\right)=0$ if and only if $Z$ and the $r+1$ linear hyperplanes $\left(\zeta_{i}, \mathbf{X}\right)=0(i=0, \ldots, r)$ have a point in common (cf. [18], pp. 65-66). From the investigations of Philippon and Soulé it follows that

$$
\left|h(Z)-\log H\left(F_{Z}\right)\right| \leq c(n) \operatorname{deg} Z,
$$

where $c(n)$ is effectively computable in terms of $n$.

Below we give an explicit version of [1], Theorem 3.3.

Theorem 2. Let $m, \mathbf{n}, \mathbf{d}, \sigma, \varepsilon, F, Z, Z_{1}, \ldots, Z_{m}, k_{0}, k_{1}, s=\sum_{h=1}^{m} \operatorname{codim} Z_{h}$ be as in Theorem 1 , except that $k=\overline{\mathbb{Q}}$. Then

$$
\begin{aligned}
{\left[k_{1}: k_{0}\right] \operatorname{deg} Z_{1} \ldots \operatorname{deg} Z_{m}\left(\sum_{h=1}^{m} \frac{1}{\operatorname{deg} Z_{h}} d_{h} h\left(Z_{h}\right)\right) } \\
\leq 2(s / \varepsilon)^{s} m^{M} M^{2}\left(d_{1}+\ldots+d_{m}+\log H(F)\right) .
\end{aligned}
$$

As mentioned in the introduction, results similar to our Theorems 1 and 2 were obtained independently by Ferretti [3].

The following corollary of Theorems 1 and 2 is useful.

Corollary. Let $m$ be an integer $\geq 2, \mathbf{n}=\left(n_{1}, \ldots, n_{m}\right), \mathbf{d}=\left(d_{1}, \ldots, d_{m}\right)$ tuples of positive integers and $\varepsilon$ a real such that $0 \leq \varepsilon \leq M+1$ and 


$$
\frac{d_{h}}{d_{h+1}} \geq\left(\frac{m M(M+1)}{\varepsilon}\right)^{M} \text { for } h=1, \ldots, m-1,
$$

where again $M:=n_{1}+\ldots+n_{m}$. Further, let $F \in \Gamma_{\bar{Q}}^{\mathbf{n}}(\mathbf{d}) \backslash\{0\}$. Then each irreducible component of $Z_{\varepsilon}$ is contained in a product variety

$$
Z_{1} \times \ldots \times Z_{m} \varsubsetneqq \mathbb{P}^{\mathbf{n}}(\overline{\mathbb{Q}}),
$$

where for $h=1, \ldots, m, Z_{h}$ is a subvariety of $\mathbb{P}^{n_{h}}(\overline{\mathbb{Q}})$. Further, if $F$ has its coefficients in an algebraic number field $k_{0}$, then $Z_{1}, \ldots, Z_{m}$ are defined over an extension $k_{1}$ of $k_{0}$ with

$$
\left[k_{1}: k_{0}\right] \operatorname{deg} Z_{1} \ldots \operatorname{deg} Z_{m} \leq\left(\frac{m(M+1) s}{\varepsilon}\right)^{s},
$$

where $s=\sum_{h=1}^{m} \operatorname{codim} Z_{h}$, and

$$
\begin{aligned}
& {\left[k_{1}: k_{0}\right] \operatorname{deg} Z_{1} \ldots \operatorname{deg} Z_{m}\left(\sum_{h=1}^{m} \frac{1}{\operatorname{deg} Z_{h}} d_{h} h\left(Z_{h}\right)\right)} \\
& \leq 2\left(\frac{(M+1) s}{\varepsilon}\right)^{s} m^{M} M^{2}\left(d_{1}+\ldots+d_{m}+\log H(F)\right) .
\end{aligned}
$$

Pro of. Put $\varepsilon^{\prime}:=\varepsilon /(M+1)$. Consider the sequence of closed subsets of $\mathbb{P}^{\mathbf{n}}(\overline{\mathbb{Q}})$ :

$$
\mathbb{P}^{\mathbf{n}}(\overline{\mathbb{Q}})=Z_{0} \supseteq Z_{\varepsilon^{\prime}} \supseteq Z_{2 \varepsilon^{\prime}} \supseteq \ldots \supseteq Z_{(M+1) \varepsilon^{\prime}}=Z_{\varepsilon} .
$$

For $i=0, \ldots, M+1$, choose an irreducible component $W_{i}$ of $Z_{i \varepsilon^{\prime}}$ such that

$$
\mathbb{P}^{\mathbf{n}}(\overline{\mathbb{Q}})=W_{0} \supseteq W_{1} \supseteq \ldots \supseteq W_{M+1}=Z \text {. }
$$

By [18], p. 54, $\mathbb{P}^{\mathbf{n}}(\overline{\mathbb{Q}})$ has dimension $n_{1}+\ldots+n_{m}=M$ and if $V_{1}, V_{2}$ are two subvarieties of $\mathbb{P}^{\mathbf{n}}(\overline{\mathbb{Q}})$ with $V_{1} \varsubsetneqq V_{2}$ then $\operatorname{dim} V_{1}<\operatorname{dim} V_{2}$. It follows that there is an $i \in\{0, \ldots, M\}$ with $W_{i}=W_{i+1}$. Clearly, $W:=W_{i}=W_{i+1} \varsubsetneqq$ $\mathbb{P}^{\mathbf{n}}(\overline{\mathbb{Q}})$ as it is contained in $\{F=0\}$. Further, $W$ is an irreducible component of both $Z_{i \varepsilon^{\prime}}$ and $Z_{i \varepsilon^{\prime}+\varepsilon^{\prime}}$. By (1.8), the conditions of Theorems 1 and 2 are satisfied with $i \varepsilon^{\prime}, \varepsilon^{\prime}$ replacing $\sigma, \varepsilon$. Hence $W=Z_{1} \times \ldots \times Z_{m}$, where $Z_{h}$ is a subvariety of $\mathbb{P}^{n_{h}}(\overline{\mathbb{Q}})$, for $h=1, \ldots, m$. Inequalities (1.9), (1.10) follow by replacing $\varepsilon$ by $\varepsilon /(M+1)$ in (1.3), (1.7), respectively.

Using the techniques of the proofs of Theorems 1 and 2 one can prove the following sharpening of a non-vanishing result of Roth from 1955 [12], now known as Roth's lemma. Roth used this in his proof of his famous theorem, also in [12], that for every algebraic number $\alpha$ and every $\kappa>2$ there are only finitely many rationals $x / y$ with $x, y \in \mathbb{Z}, y>0$ and $|\alpha-x / y|<y^{-\kappa}$. In fact, from the Corollary with $n_{1}=\ldots=n_{m}=1$ one can derive Theorem 3 below with instead of (1.11) the more restrictive condition $d_{h} / d_{h+1} \geq\left(2 m^{3} / \varepsilon\right)^{m}$ for $h=1, \ldots, m-1$. 
Theorem 3 (Roth's lemma). Let $m$ be an integer $\geq 2$, let $\mathbf{d}=\left(d_{1}, \ldots\right.$ $\left.\ldots, d_{m}\right)$ be a tuple of positive integers, let $F \in \overline{\mathbb{Q}}\left[X_{10}, X_{11} ; \ldots ; X_{m 0}, X_{m 1}\right]$ be a non-zero polynomial which is homogeneous of degree $d_{h}$ in the pair of variables $\left(X_{h 0}, X_{h 1}\right)$ for $h=1, \ldots, m$ and let $\varepsilon$ be a real with $0<\varepsilon \leq m+1$ such that

$$
d_{h} / d_{h+1} \geq 2 m^{3} / \varepsilon \quad \text { for } h=1, \ldots, m-1 .
$$

Further, let $P=\left(P_{1}, \ldots, P_{m}\right)$, where $P_{1}, \ldots, P_{m}$ are points in $\mathbb{P}^{1}(\overline{\mathbb{Q}})$ with

$$
H\left(P_{h}\right)^{d_{h}}>\left\{e^{d_{1}+\ldots+d_{m}} H(F)\right\}^{\left(3 m^{3} / \varepsilon\right)^{m}} \quad \text { for } h=1, \ldots, m,
$$

where $e=2.7182 \ldots$ Then $i_{\mathbf{d}}(F, P)<\varepsilon$.

The original lemma proved by Roth in 1955 [12] differs from Theorem 3 in that instead of (1.11) it has the more restrictive condition

$$
d_{h} / d_{h+1} \geq\left(10^{m} / \varepsilon\right)^{2^{m}} \quad \text { for } h=1, \ldots, m-1 .
$$

Roth's lemma with (1.13) was also used by Schmidt in his proof of the Subspace Theorem and by Schmidt and Schlickewei in their proofs of quantitative versions of the Subspace Theorem. In our improvements of the results of Schmidt and Schlickewei mentioned in the introduction, it was crucial that (1.13) could be replaced by (1.11).

R e mark (inspired by a suggestion of the referee). We have formulated the Product Theorem and its consequences for multi-homogeneous polynomials. There are affine analogues for polynomials which are not multihomogeneous. For instance, for $h=1, \ldots, m$, let $\mathbf{Y}_{h}=\left(Y_{h 1}, \ldots, Y_{h, n_{h}}\right)$ be a block of affine variables, and let $f \in k\left[\mathbf{Y}_{1}, \ldots, \mathbf{Y}_{m}\right]$ be a polynomial whose total degree in the block $\mathbf{Y}_{h}$ is at most $d_{h}$, for $h=1, \ldots, m$. Denote by $\mathbf{i}$ and $\mathbf{k}$ tuples $\left(i_{h j}: h=1, \ldots, m, j=0, \ldots, n_{h}\right)$ and $\left(k_{h j}\right.$ : $\left.h=1, \ldots, m, j=1, \ldots, n_{h}\right)$, respectively. Define the index of $f$ at a point $p$ as the largest number $\sigma$ such that $f_{\mathbf{k}}(p)=0$ for all tuples $\mathbf{k}$ with $\sum_{h=1}^{m} d_{h}^{-1}\left(k_{h 1}+\ldots+k_{h, n_{h}}\right) \leq \sigma$, where $f_{\mathbf{k}}=\left(\prod_{h=1}^{m} \prod_{j=1}^{n_{h}} \partial^{k_{h j}} / \partial Y_{h j}^{k_{h j}}\right) f$. For $h=1, \ldots, m$, define a block of variables $\mathbf{X}_{h}=\left(X_{h 0}, \ldots, X_{h m}\right)$ such that $Y_{h j}=X_{h j} / X_{h 0}$ for $j=1, \ldots, n_{h}$. Let $F=\prod_{h=1}^{m} X_{h 0}^{d_{h}} f$ be the multihomogeneous polynomial in $\mathbf{X}_{1}, \ldots, \mathbf{X}_{m}$ corresponding to $f$. One obtains an analogue of Theorem 1 for $f$ (the same statement with everywhere "affine varieties" replacing "projective varieties") simply by applying Theorem 1 to $F$.

We have to check that the index of $f$ at $p=\left(p_{11}, \ldots, p_{1, n_{1}} ; \ldots ; p_{m 1}, \ldots\right.$, $\left.p_{m, n_{m}}\right)$, defined using the variables $Y_{h j}$, is equal to the index of $F$ at $P=$ $\left(1, p_{11}, \ldots, p_{1, n_{1}} ; \ldots ; 1, \ldots, p_{m, n_{m}}\right)$ defined using the variables $X_{h j}$. This follows by observing first that $f_{\mathbf{k}}=H^{-1} F_{\mathbf{i}}$, where $H$ is a product of powers of $X_{h 0}(h=1, \ldots, m)$ and $\mathbf{i}$ is the same tuple as $\mathbf{k}$ augmented with $i_{h 0}:=0$ for $h=1, \ldots, m$, and second, in view of Euler's identity $\partial H / \partial X_{h 0}=$ 
$X_{h 0}^{-1}\left(e_{h} H-\sum_{j=1}^{n_{h}} X_{h j} \partial H / \partial X_{h j}\right)$ for polynomials $H$ homogeneous of degree $e_{h}$ in $\mathbf{X}_{h}$, that for each tuple $\mathbf{i}, F_{\mathbf{i}}$ is a linear combination of $f_{\mathbf{k}}$ over tuples $\mathbf{k}$ with $k_{h j} \leq i_{h j}$ for $h=1, \ldots, m, j=1, \ldots, n_{h}$, the coefficients being rational functions whose denominators are products of powers of $X_{h 0}(h=1, \ldots, m)$.

2. Intersection theory. Most of the results from intersection theory we need can be found in [4], Chaps. 1, 2 and in [9]. As in Section 1, $k$ denotes an algebraically closed field and $\mathbf{n}=\left(n_{1}, \ldots, n_{m}\right)$ a tuple of positive integers. The block $\mathbf{X}_{h}$ of $n_{h}+1$ variables, the ring $k[\mathbf{X}]=k\left[\mathbf{X}_{1}, \ldots, \mathbf{X}_{m}\right]$ and the sets $\Gamma_{k}^{\mathbf{n}}(\mathbf{d})$ will have the meaning of Section 1 . We write $\mathbb{P}^{\mathbf{n}}, \Gamma^{\mathbf{n}}, \Gamma^{\mathbf{n}}(\mathbf{d})$ for $\mathbb{P}^{\mathbf{n}}(k), \Gamma_{k}^{\mathbf{n}}, \Gamma_{k}^{n}(\mathbf{d})$.

For every subvariety $Z$ of $\mathbb{P}^{\mathbf{n}}$ there is a unique essential $\mathbf{n}$-prime ideal $I$ of $k[\mathbf{X}]$ such that $Z=V(I)=\left\{P \in \mathbb{P}^{\mathbf{n}}: f(P)=0\right.$ for every $\left.f \in I\right\}$. The local ring of $Z$ is defined by

$$
\mathcal{O}_{Z}:=\left\{f / g: \exists \mathbf{d} \in\left(\mathbb{Z}_{\geq 0}\right)^{m} \text { with } f, g \in \Gamma^{\mathbf{n}}(\mathbf{d}), g \notin I\right\} .
$$

For any n-ideal $J$ of $k[\mathbf{X}]$ we put $J \mathcal{O}_{Z}:=\left\{f / g: \exists \mathbf{d} \in\left(\mathbb{Z}_{>0}\right)^{m}\right.$ with $f, g \in$ $\left.\Gamma^{\mathbf{n}}(\mathbf{d}), f \in J, g \notin I\right\}$. Then $M_{Z}:=I \mathcal{O}_{Z}$ is the maximal ideal of $\mathcal{O}_{Z}$. The residue field $k(Z):=\mathcal{O}_{Z} / M_{Z}$ is called the function field of $Z$. The dimension of $Z$ is $\operatorname{dim} Z:=\operatorname{trdeg}_{k} k(Z)$. In particular, $\operatorname{dim} \mathbb{P}^{\mathbf{n}}=M:=n_{1}+\ldots+n_{m}$. The codimension of $Z$ is $\operatorname{codim} Z:=M-\operatorname{dim} Z$; if $W$ is a subvariety of $Z$ then the codimension of $W$ in $Z$ is $\operatorname{codim}(W, Z)=\operatorname{dim} Z-\operatorname{dim} W$.

A cycle in $\mathbb{P}^{\mathbf{n}}$ is a finite formal linear combination with integer coefficients of subvarieties $V$ of $\mathbb{P}^{\mathbf{n}}, Z=\sum n_{V} V$, say. The components of $Z$ are the subvarieties $V$ for which $n_{V} \neq 0$, and $n_{V}$ is called the multiplicity of $V$ in $Z$. $Z$ is called effective if all $n_{V} \geq 0$. Denote by $\mathcal{Z}_{k}=\mathcal{Z}_{k}\left(\mathbb{P}^{\mathbf{n}}\right)$ the abelian group of cycles in $\mathbb{P}^{\mathbf{n}}$ all of whose components have dimension $k$ and put $\mathcal{Z}_{k}:=(0)$ for $k<0$. We denote by $Z$ cycles as well as varieties.

For a ring $A$ and an $A$-module $M$, we define the length $l_{A}(M)$ to be the integer $l$ for which there exists a sequence of $A$-modules

$$
M=M_{0} \supseteq M_{1} \supseteq \ldots \nsupseteq M_{l}=(0)
$$

such that $M_{i-1} / M_{i} \cong A / p_{i}$ for $i=1, \ldots, l$, where $p_{i}$ is a maximal ideal of $A$ (cf. [4], p. 406); $l_{A}(M)$ is independent of the choice of $M_{0}, \ldots, M_{l}$. Now let $Z=V(I)$ be a subvariety of $\mathbb{P}^{\mathbf{n}}$ and $f \in \Gamma^{\mathbf{n}} \backslash\{0\}$ such that $f$ does not vanish identically on $Z$, i.e. $f \notin I$. We define the divisor of $f$ restricted to $Z$ by attaching certain multiplicities to the irreducible components of $Z \cap\{f=0\}$. These irreducible components are all of codimension 1 in $V$ (cf. [21], p. 196). For each subvariety $W$ of $Z$ with $\operatorname{codim}(W, Z)=1$, the number

$$
\operatorname{ord}_{W}(f \mid Z):=l_{\mathcal{O}_{W}}\left(\mathcal{O}_{W} /(I+(f)) \mathcal{O}_{W}\right)
$$


is a finite, non-negative integer and $\operatorname{ord}_{W}(f \mid Z)>0$ if and only if $I+(f)$ is contained in the prime ideal of $W$, i.e. if $W$ is an irreducible component of $Z \cap\{f=0\}$. Now define

$$
\operatorname{div}(f \mid Z)=\sum_{W} \operatorname{ord}_{W}(f \mid Z) \cdot W,
$$

where the sum is taken over all subvarieties $W$ of codimension 1 in $Z$. By [3], App. A3, $\operatorname{ord}_{W}(f g \mid Z)=\operatorname{ord}_{W}(f \mid Z)+\operatorname{ord}_{W}(g \mid Z)$ and hence $\operatorname{div}(f g \mid Z)=$ $\operatorname{div}(f \mid Z)+\operatorname{div}(g \mid Z)$ whenever $f, g$ do not identically vanish on $Z$. By abuse of terminology, we say that $f$ does not identically vanish on a cycle $Z=$ $\sum n_{V} V$ if for each component $V$ of $Z, f$ does not identically vanish on $V$. In that case we $\operatorname{define} \operatorname{div}(f \mid Z)=\sum n_{V} \operatorname{div}(f \mid V)$. Note that $\operatorname{div}(f \mid Z)$ is effective if $Z$ is effective. We write $\operatorname{div}(f)$ if $Z=\mathbb{P}^{\mathbf{n}}$.

Two cycles $Z_{1}, Z_{2} \in \mathcal{Z}_{t}\left(\mathbb{P}^{\mathbf{n}}\right)$ are called rationally equivalent if $Z_{1}-Z_{2}$ is a linear combination of cycles $\operatorname{div}(f \mid V)-\operatorname{div}(g \mid V)$, where $V$ is a $(t+$ 1)-dimensional subvariety of $\mathbb{P}^{\mathbf{n}}$ and $f, g \in \Gamma^{\mathbf{n}}(\mathbf{d})$ for some $\mathbf{d} \in\left(\mathbb{Z}_{>0}\right)^{n}$. Addition of cycles induces addition of rational equivalence classes. Note that all divisors $\operatorname{div}(f)$ with $f \in \Gamma^{\mathbf{n}}(\mathbf{d})\left(\mathbf{d} \in\left(\mathbb{Z}_{\geq 0}\right)^{m}\right)$ are rationally equivalent; denote by $\mathcal{O}(\mathbf{d})$ the rational equivalence class of $\operatorname{div}(f), f \in \Gamma^{\mathbf{n}}(\mathbf{d})$. Clearly, $\mathcal{O}\left(\mathbf{d}_{1}\right)+\mathcal{O}\left(\mathbf{d}_{2}\right)=\mathcal{O}\left(\mathbf{d}_{1}+\mathbf{d}_{2}\right)$. We define $\mathcal{O}(\mathbf{d})$ for $\mathbf{d} \in \mathbb{Z}^{m}$ by additivity. Put $\operatorname{Pic}(\mathbf{n})=\left\{\mathcal{O}(\mathbf{d}): \mathbf{d} \in \mathbb{Z}^{m}\right\}, \operatorname{Pic}^{+}(\mathbf{n})=\left\{\mathcal{O}(\mathbf{d}): \mathbf{d} \in\left(\mathbb{Z}_{\geq 0}\right)^{m}\right\}$. If $\mathcal{M}=\mathcal{O}(\mathbf{d}) \in \operatorname{Pic}^{+}(\mathbf{n})$, then write $\Gamma(\mathcal{M})$ or $\Gamma_{k}(\mathcal{M})$ for $\Gamma_{k}^{\mathbf{n}}(\mathbf{d})$.

For a zero-dimensional cycle $Z=\sum_{P} n_{P} P$ we define its degree:

$$
\operatorname{deg} Z:=\sum_{P} n_{P} .
$$

Then we have:

LEMma 1. For $t=0, \ldots, M$ there is a unique function (intersection number $)$ from $\mathcal{Z}_{t}\left(\mathbb{P}^{\mathbf{n}}\right) \times \operatorname{Pic}(\mathbf{n})^{t}$ to $\mathbb{Z}:\left(Z, \mathcal{M}_{1}, \ldots, \mathcal{M}_{t}\right) \mapsto\left(Z \cdot \mathcal{M}_{1} \ldots \mathcal{M}_{t}\right)$ with the following properties:

(i) $\left(Z \cdot \mathcal{M}_{1} \ldots \mathcal{M}_{t}\right)$ is additive in $Z, \mathcal{M}_{1}, \ldots, \mathcal{M}_{t}$ and invariant under permutations of $\mathcal{M}_{1}, \ldots, \mathcal{M}_{t}$;

(ii) $\left(Z \cdot \mathcal{M}_{1} \ldots \mathcal{M}_{t}\right)=0$ if $Z$ is rationally equivalent to 0 ;

(iii) if $Z \in \mathcal{Z}_{0}\left(\mathbb{P}^{\mathbf{n}}\right)$ then $(Z)=\operatorname{deg} Z$;

(iv) if $\mathcal{M}_{1} \in \operatorname{Pic}^{+}(\mathbf{n})$ then there is an $f \in \Gamma\left(\mathcal{M}_{1}\right)$ not identically vanishing on $Z$ and for every such $f$ we have

$$
\left(Z \cdot \mathcal{M}_{1} \ldots \mathcal{M}_{t}\right)=\left(\operatorname{div}(f \mid Z) \cdot \mathcal{M}_{2} \ldots \mathcal{M}_{t}\right) .
$$

Proof. This comprises some of the results from [4], Chaps. 1, 2. Rationally equivalent cycles in $\mathcal{Z}_{0}$ have the same degree and if $Z, Z^{\prime} \in \mathcal{Z}_{t}$ are rationally equivalent and $f, f^{\prime} \in \Gamma\left(\mathcal{M}_{1}\right)$, then $\operatorname{div}(f \mid Z), \operatorname{div}\left(f \mid Z^{\prime}\right)$ are rationally equivalent. Hence the intersection number can be defined inductively by (iii), (iv). 
We write $\left(\mathcal{M}_{1} \ldots \mathcal{M}_{m}\right)$ for $\left(\mathbb{P}^{\mathbf{n}} \cdot \mathcal{M}_{1} \ldots \mathcal{M}_{m}\right)$. Further, for divisor classes $\mathcal{N}_{1}, \ldots, \mathcal{N}_{s}$ and non-negative integers $e_{1}, \ldots, e_{s}$ with $e_{1}+\ldots+e_{s}=t$, we write $\left(Z \cdot \mathcal{N}_{1}^{e_{1}} \ldots \mathcal{N}_{s}^{e_{s}}\right)$ for $(Z \cdot \underbrace{\mathcal{N}_{1} \ldots \mathcal{N}_{1}}_{e_{1} \text { times }} \cdots \underbrace{\mathcal{N}_{s} \ldots \mathcal{N}_{s}}_{e_{s} \text { times }})$. The degree of $Z \in \mathcal{Z}_{t}$ is defined by $\operatorname{deg} Z:=\left(Z \cdot \mathcal{O}(1)^{t}\right)$.

Remarks. (i) By induction on the dimension it follows easily that if $Z \in \mathcal{Z}_{t}$ is effective and $\mathcal{M}_{1}, \ldots, \mathcal{M}_{t} \in \operatorname{Pic}^{+}(\mathbf{n})$ then $\left(Z \cdot \mathcal{M}_{1} \ldots \mathcal{M}_{t}\right) \geq 0$. Moreover, if $Z$ is a subvariety of $\mathbb{P}^{\mathbf{n}}$ and $f_{1} \in \Gamma\left(\mathcal{M}_{1}\right), \ldots, f_{t} \in \Gamma\left(\mathcal{M}_{t}\right)$ are "generic", then $\left(Z \cdot \mathcal{M}_{1} \ldots \mathcal{M}_{t}\right)$ is precisely the cardinality of the set of points $V \cap\left\{f_{1}=\ldots=f_{t}=0\right\}$.

(ii) Let $k_{0}$ be a perfect subfield of $k$, i.e. every finite extension of $k_{0}$ is separable. A $k_{0}$-subvariety of $\mathbb{P}^{\mathbf{n}}$ is a set $\left\{P \in \mathbb{P}^{\mathbf{n}}: f(P)=0\right.$ for every $\left.f \in I\right\}$, where $I$ is an essential $\mathbf{n}$-prime ideal of $k_{0}[\mathbf{X}]$. Every such $k_{0}$-subvariety $Z$ is a union of equal dimensional subvarieties of $\mathbb{P}^{\mathbf{n}}, Z=Z_{1} \cup \ldots \cup Z_{q}$, and we put $\operatorname{dim} Z:=\operatorname{dim} Z_{1}$; now if $\operatorname{dim} Z=k$ and $\mathcal{M}_{1}, \ldots, \mathcal{M}_{t} \in \operatorname{Pic}^{+}(\mathbf{n})$ then we define

$$
\left(Z \cdot \mathcal{M}_{1} \ldots \mathcal{M}_{t}\right):=\sum_{i=1}^{q}\left(Z_{i} \cdot \mathcal{M}_{1} \ldots \mathcal{M}_{t}\right) .
$$

This is extended by linearity to $k_{0^{-}}$cycles, i.e. finite formal sums of $k_{0^{-}}$ subvarieties.

We need some further properties of the intersection number. Let $\mathbf{e}_{1}=$ $(1,0, \ldots, 0), \mathbf{e}_{2}=(0,1, \ldots, 0), \ldots, \mathbf{e}_{m}=(0, \ldots, 0,1)$ and put $\mathcal{L}_{h}=\mathcal{O}\left(\mathbf{e}_{h}\right)$ for $h=1, \ldots, m$. Further, fix $\mathbf{d}=\left(d_{1}, \ldots, d_{m}\right) \in\left(\mathbb{Z}_{>0}\right)^{m}$ and put $\mathcal{L}:=$ $\mathcal{O}(\mathbf{d})=d_{1} \mathcal{L}_{1}+\ldots+d_{m} \mathcal{L}_{m}$. If $Z_{h}=\sum_{V_{h}} n_{V_{h}} V_{h}(h=1, \ldots, m)$ is a cycle in $\mathbb{P}^{n_{h}}$ then of course we define

$$
Z_{1} \times \ldots \times Z_{m}=\sum n_{V_{1}} n_{V_{2}} \ldots n_{V_{m}} V_{1} \times \ldots \times V_{m} .
$$

Further, we denote by $\pi_{h}$ the projection to the $h$ th factor $\mathbb{P}^{\mathbf{n}} \rightarrow \mathbb{P}^{n_{h}}$ and by $\pi_{h}^{*}$ the inclusion ("pull back") $k\left[\mathbf{X}_{h}\right] \hookrightarrow k\left[\mathbf{X}_{1}, \ldots, \mathbf{X}_{m}\right]=k[\mathbf{X}]$.

Lemma 2. Let $Z_{h} \in \mathcal{Z}_{\delta_{h}}\left(\mathbb{P}^{n_{h}}\right)(h=1, \ldots, m)$ and $Z=Z_{1} \times \ldots \times Z_{m}$. Put $\delta=\delta_{1}+\ldots+\delta_{m}$.

(i) Suppose that $f \in \Gamma^{n_{1}}$ does not vanish identically on $Z_{1}$. Then $\pi_{1}^{*} f$ does not vanish identically on $Z$ and

$$
\operatorname{div}\left(\pi_{1}^{*} f \mid Z\right)=\operatorname{div}\left(f \mid Z_{1}\right) \times Z_{2} \times \ldots \times Z_{m} .
$$

(ii) Let $e_{1}, \ldots, e_{m}$ be non-negative integers with $e_{1}+\ldots+e_{m}=\delta$. Then

$$
\left(Z \cdot \mathcal{L}_{1}^{e_{1}} \ldots \mathcal{L}_{m}^{e_{m}}\right)= \begin{cases}\operatorname{deg} Z_{1} \ldots \operatorname{deg} Z_{m} & \text { if }\left(e_{1}, \ldots, e_{m}\right)=\left(\delta_{1}, \ldots, \delta_{m}\right), \\ 0 & \text { otherwise. }\end{cases}
$$


(iii) $\left(Z \cdot \mathcal{L}^{\delta}\right)=\left(\delta ! /\left(\delta_{1} ! \ldots \delta_{m} !\right)\right) d_{1}^{\delta_{1}} \ldots d_{m}^{\delta_{m}} \operatorname{deg} Z_{1} \ldots \operatorname{deg} Z_{m}$. In particular $\left(\mathcal{L}^{M}\right)=C:=\left(M ! /\left(n_{1} ! \ldots n_{m} !\right)\right) d_{1}^{n_{1}} \ldots d_{m}^{n_{m}}$.

Proof. (i) ([4], p. 35, Ex. 2.3.1). This is analogous to the set-theoretic statement that if $Z_{1}, \ldots, Z_{m}$ are varieties then $Z \cap\left\{\pi_{1}^{*} f=0\right\}=\left(Z_{1} \cap\{f=\right.$ $0\}) \times Z_{2} \times \ldots \times Z_{m}$.

(ii) This follows easily from (i) by induction on $\delta$. Another way is as follows. For $h=1, \ldots, m$ assume that $Z_{h}$ is a subvariety of $\mathbb{P}^{n_{h}}$, take generic linear forms $f_{h j} \in k\left[\mathbf{X}_{h}\right]$ for $j=1, \ldots, e_{h}$ and put $W_{h}=Z_{h} \cap\left\{f_{h 1}=\right.$ $\left.0, \ldots, f_{h, e_{h}}=0\right\}$. Then by remark (i) above $\left(Z \cdot \mathcal{L}_{1}^{e_{1}} \ldots \mathcal{L}_{m}^{e_{m}}\right)$ is the cardinality of the set $W=W_{1} \times \ldots \times W_{m}$. This cardinality is zero if $\left(e_{1}, \ldots, e_{m}\right) \neq$ $\left(\delta_{1}, \ldots, \delta_{m}\right)$ since then one of the sets $W_{h}$ is empty; while otherwise this cardinality is $\prod_{h=1}^{m} \# W_{h}=\prod_{h=1}^{m} \operatorname{deg} Z_{h}$.

(iii) By additivity we have

$$
\begin{aligned}
\left(Z \cdot \mathcal{L}^{\delta}\right) & =\left(Z \cdot\left(d_{1} \mathcal{L}_{1}+\ldots+d_{m} \mathcal{L}_{m}\right)^{\delta}\right) \\
& =\sum_{e_{1}+\ldots+e_{m}=d} \frac{\delta !}{e_{1} ! \ldots e_{m} !} d_{1}^{e_{1}} \ldots d_{m}^{e_{m}}\left(Z \cdot \mathcal{L}_{1}^{e_{1}} \ldots \mathcal{L}_{m}^{e_{m}}\right) \\
& =\frac{\delta !}{\delta_{1} ! \ldots \delta_{m} !} d_{1}^{\delta_{1}} \ldots d_{m}^{\delta_{m}} \operatorname{deg} Z_{1} \ldots \operatorname{deg} Z_{m} .
\end{aligned}
$$

Lemma 3. Suppose that $m \geq 2$. Let $Z$ be a $\delta$-dimensional subvariety of $\mathbb{P}^{\mathbf{n}}$ that cannot be expressed as a product $Z=Z_{1} \times \ldots \times Z_{m}$ with $Z_{h} \subseteq \mathbb{P}^{n_{h}}$ for $h=1, \ldots, m$. Then there are at least two tuples of non-negative integers $\left(e_{1}, \ldots, e_{m}\right)$ with $e_{1}+\ldots+e_{m}=\delta$ and $\left(Z \cdot \mathcal{L}_{1}^{e_{1}} \ldots \mathcal{L}_{m}^{e_{m}}\right)>0$.

Proof (cf. [11], p. 79). The idea is as follows. By [18], p. 45, Thm. 2, if $X$ is a closed subset of $\mathbb{P}^{\mathbf{n}}$ and $f: X \rightarrow \mathbb{P}^{\mathbf{n}}$ a morphism, then $f(X)$ is closed, and $f$ maps subvarieties of $X$ to subvarieties of $f(X)$. We apply this with the projections $\pi_{h}: \mathbb{P}^{\mathbf{n}} \rightarrow \mathbb{P}^{n_{h}}$. Put $Z_{h}:=\pi_{h}(Z), \delta_{h}:=\operatorname{dim} Z_{h}$ for $h=1, \ldots, m$. Since $Z$ is not a product, $Z$ is a proper subvariety of $Z_{1} \times \ldots \times Z_{m}$ and therefore, $\delta=\operatorname{dim} Z<\operatorname{dim} Z_{1} \times \ldots \times Z_{m}=\delta_{1}+\ldots+\delta_{m}$. We prove by induction on $m$ the following assertion: for each $h \in\{1, \ldots, m\}$ there is a tuple $\left(e_{1}, \ldots, e_{m}\right)$ as in the statement of Lemma 3 with $e_{h}=\delta_{h}$. This implies Lemma 3 since $\delta_{1}+\ldots+\delta_{m}>\delta$.

This assertion is obviously true if $m=1$. Suppose that the assertion holds for $m=r-1$ where $r>1$. We prove the assertion for $m=r, h=1$, which clearly suffices. In the induction step we proceed by induction on $\delta_{1}$. If $\delta_{1}=0$ then $Z=Q \times W$ where $Q \in \mathbb{P}^{n_{1}}$ and $W$ is a subvariety of $\mathbb{P}^{n_{2}} \times \ldots \times \mathbb{P}^{n_{m}}$ and the assertion follows by applying the induction hypothesis to $W$. If $\delta_{1}>0$ then choose a linear form $f \in k\left[\mathbf{X}_{1}\right]$ that does not identically vanish on $Z_{1}$. Then $g:=\pi_{1}^{*} f$ does not identically vanish on $Z$. Clearly, $\pi_{1}$ maps the irreducible components of $Z \cap\{g=0\}$ to those of $Z_{1} \cap\{f=0\}$ and the latter have dimension $\delta_{1}-1$. By applying the second induction 
hypothesis to the irreducible components of $Z \cap\{g=0\}$, we infer that there are non-negative integers $e_{1}, \ldots, e_{m}$ with $e_{1}+\ldots+e_{m}=\delta$ and $e_{1}=\delta_{1}$ such that $\left(\operatorname{div}(g \mid Z) \cdot \mathcal{L}_{1}^{e_{1}-1} \ldots \mathcal{L}_{m}^{e_{m}}\right)>0$. Hence $\left(Z \cdot \mathcal{L}_{1}^{e_{1}} \ldots \mathcal{L}_{m}^{e_{m}}\right)>0$. This proves the assertion.

LEMma 4. Let $A$ be a set of polynomials from $\Gamma^{\mathbf{n}}(\mathbf{d}) \backslash\{0\}$ and I the ideal generated by $A$. Let $Z_{1}, \ldots, Z_{t}$ be irreducible components of codimension $t$ of $X:=\left\{P \in \mathbb{P}^{\mathbf{n}}: f(P)=0\right.$ for $\left.f \in A\right\}$. Then for all tuples of non-negative integers $\left(e_{1}, \ldots, e_{m}\right)$ with $e_{1}+\ldots+e_{m}=M-t$ one has

$$
\sum_{i=1}^{r} m_{Z_{i}}\left(Z_{i} \cdot \mathcal{L}_{1}^{e_{1}} \ldots \mathcal{L}_{m}^{e_{m}}\right) \leq\left(\mathcal{L}_{1}^{e_{1}} \ldots \mathcal{L}_{m}^{e_{m}} \cdot \mathcal{L}^{t}\right)
$$

where $m_{Z_{i}}:=l_{\mathcal{O}_{Z_{i}}}\left(\mathcal{O}_{Z_{i}} / I \mathcal{O}_{Z_{i}}\right)$ for $i=1, \ldots, r$.

Proof. This is essentially Proposition 2.3 of [1] and Lemma 6.4, p. 76 of [9]. We give some details of the proof to which we have to refer later. For a subvariety $Z$ of $\mathbb{P}^{\mathbf{n}}$ and $f \in \Gamma^{\mathbf{n}} \backslash\{0\}$ not vanishing identically on $Z$, define the truncated divisor

$$
\operatorname{div}^{X}(f \mid Z):=\sum_{W \not \subset X} \operatorname{ord}_{W}(f \mid Z) W,
$$

where the sum is taken over all irreducible components of $\operatorname{div}(f \mid Z)$ which are not irreducible components of $X$. This is extended by linearity to cycles. Put $Z_{0}:=\mathbb{P}^{\mathbf{n}}$ and choose inductively $f_{1}, \ldots, f_{t} \in I$ and define cycles $C_{1}, \ldots, C_{t}$ as follows:

(2.6) for $j=1, \ldots, t, f_{j}$ does not vanish identically on $C_{j-1}$, each $Z_{i}$ $(i=1, \ldots, r)$ is a subvariety of one of the irreducible components of $\operatorname{div}\left(f_{j} \mid C_{j-1}\right)$, and $C_{j}:=\operatorname{div}^{X}\left(f_{j} \mid C_{j-1}\right)$;

in the next lemma we explicitly construct such $f_{j}$. Clearly, the irreducible components of $C_{j}$ have codimension $j$. Therefore $Z_{1}, \ldots, Z_{r}$ are irreducible components of $C_{t}$.

We need some more advanced results from intersection theory to estimate the multiplicity $m_{Z_{i}, C_{t}}$ of $Z_{i}$ in $C_{t}$ from below. By [4], Ex. 7.1.10, p. 123, $m_{Z_{i}, C_{t}}$ is equal to $l_{\mathcal{O}_{Z_{i}}}\left(\mathcal{O}_{Z_{i}} / I^{\prime} \mathcal{O}_{Z_{i}}\right)$, where $I^{\prime}=\left(f_{1}, \ldots, f_{t}\right)$. (Note that $\mathbb{P}^{\mathbf{n}}$ is smooth whence all local rings $\mathcal{O}_{Z_{i}}$ are Cohen-Macaulay rings.) Further, since $I^{\prime} \subseteq I$ we have $l_{\mathcal{O}_{Z_{i}}}\left(\mathcal{O}_{Z_{i}} / I^{\prime} \mathcal{O}_{Z_{i}}\right) \geq l_{\mathcal{O}_{Z_{i}}}\left(\mathcal{O}_{Z_{i}} / I \mathcal{O}_{Z_{i}}\right)=m_{Z_{i}}$. Hence $m_{Z_{i}, C_{t}} \geq m_{Z_{i}}$. It follows that

$$
C_{t}=\sum_{i=1}^{r} m_{Z_{i}} Z_{i}+(\text { effective cycle }) .
$$

Further, by (2.6) we have

$$
\operatorname{div}\left(f_{j} \mid C_{j-1}\right)=C_{j}+(\text { effective cycle }) \quad \text { for } j=1, \ldots, t .
$$


Now by $(2.7),(2.8)$ and $f_{j} \in \Gamma(\mathcal{L})$ we have

$$
\begin{aligned}
\sum_{i=1}^{r} m_{Z_{i}}\left(Z_{i} \cdot \mathcal{L}_{1}^{e_{1}} \ldots \mathcal{L}_{m}^{e_{m}}\right) & \leq\left(C_{t} \cdot \mathcal{L}_{1}^{e_{1}} \ldots \mathcal{L}_{m}^{e_{m}}\right) \\
& \leq\left(C_{t-1} \cdot \mathcal{L} \cdot \mathcal{L}_{1}^{e_{1}} \ldots \mathcal{L}_{m}^{e_{m}}\right) \\
& \leq\left(C_{t-2} \cdot \mathcal{L}^{2} \cdot \mathcal{L}_{1}^{e_{1}} \ldots \mathcal{L}_{m}^{e_{m}}\right) \\
& \leq \ldots \leq\left(\mathcal{L}^{t} \cdot \mathcal{L}_{1}^{e_{1}} \ldots \mathcal{L}_{m}^{e_{m}}\right) .
\end{aligned}
$$

Lemma 5. It is possible to choose $f_{1}, \ldots, f_{t}$ as in (2.6) such that

$$
f_{i}=\sum_{j=1}^{C} n_{i j} g_{i j} \quad \text { for } i=1, \ldots, t,
$$

where $C=\left(M ! /\left(n_{1} ! \ldots n_{m} !\right)\right) d_{1}^{n_{1}} \ldots d_{m}^{n_{m}}$ and $g_{i j} \in A, n_{i j} \in \mathbb{Z},\left|n_{i j}\right| \leq C$ for $i=1, \ldots, t, j=1, \ldots, C$.

Proof. We have $\left(C_{i} \cdot \mathcal{L}^{M-i}\right) \leq\left(C_{i-1} \cdot \mathcal{L}^{M-i+1}\right)$ for $i=1, \ldots, t$ by $(2.6)$ and Lemma 1. Hence

$$
\left(C_{i} \cdot \mathcal{L}^{M-i}\right) \leq\left(\mathcal{L}^{M}\right)=C \quad \text { for } i=1, \ldots, t .
$$

Letting $C_{i}=\sum_{k=1}^{u} a_{k} V_{k}$, where the $V_{k}$ are the components of $C_{i}$ and $a_{k}>0$, we see that $u \leq \sum_{k=1}^{u} a_{k}\left(V_{k} \cdot \mathcal{L}^{M-i}\right)=\left(C_{i} \cdot \mathcal{L}^{M-i}\right) \leq C$. Hence each $C_{i}$ has at most $C$ irreducible components.

Suppose we have already chosen $f_{1}, \ldots, f_{s}(0 \leq s \leq t-1)$ such that (2.6) and $(2.9)$ are satisfied for $i=1, \ldots, s$. Let $V_{1}, \ldots, V_{u^{\prime}}$ be the components of $C_{s}$ which are not irreducible components of $X$. Then for $j=1, \ldots, u^{\prime}$, there is a $g_{j} \in A$ which does not vanish identically on $V_{j}$. We construct $h_{1}, \ldots, h_{u^{\prime}}$ such that for $j=1, \ldots, u^{\prime}, h_{j}$ is not identically zero on $V_{1}, \ldots, V_{j}$ inductively as follows: Take $h_{1}=g_{1}$. Suppose that $h_{j}$ has been constructed. There are $\mathbf{x}_{1} \in V_{1}, \ldots, \mathbf{x}_{j} \in V_{j}$ such that $h_{j}\left(\mathbf{x}_{i}\right) \neq 0$ for $i=1, \ldots, j$; further, there is $\mathbf{x}_{j+1} \in V_{j+1}$ with $g_{j+1}\left(\mathbf{x}_{j+1}\right) \neq 0$. Now there is an $a \in\left\{0, \ldots, u^{\prime}\right\}$ with $\left(h_{j}+a g_{j+1}\right)\left(\mathbf{x}_{i}\right) \neq 0$ for $i=1, \ldots, j+1$; take $h_{j+1}:=h_{j}+a g_{j+1}$. Obviously, $f_{s+1}:=h_{u^{\prime}}$ does not identically vanish on $C_{s}$ and $f_{1}, \ldots, f_{s+1}$ satisfy $(2.6),(2.9)$. By repeating this process we arrive at $f_{1}, \ldots, f_{t}$ satisfying $(2.6),(2.9)$.

3. The Faltings height. From [1] we have collected some properties of the Faltings height of varieties over $\overline{\mathbb{Q}}$. We use the following notation. The extension of a ring homomorphism $\psi: R_{1} \rightarrow R_{2}$ to $R_{1}\left[X_{1}, \ldots, X_{t}\right] \rightarrow$ $R_{2}\left[X_{1}, \ldots, X_{t}\right]$, defined by applying $\psi$ to the coefficients of $f \in R_{1}\left[X_{1}, \ldots\right.$ $\left.\ldots, X_{t}\right]$, is denoted also by $\psi$. The ring of integers of a number field $K$ is denoted by $O_{K}$. For a non-zero prime ideal $\wp$ of $O_{K}$, let

$$
\mathbb{F}_{\wp}:=O_{K} / \wp, \quad N_{\wp}:=\# \mathbb{F}_{\wp},
$$


and let $\overline{\mathbb{F}}_{\wp}$ denote the algebraic closure of $\mathbb{F}_{\wp}$. The multi-projective space $\mathbb{P}^{\mathbf{n}}(\mathbb{C})$, as well as its algebraic subvarieties, can be given the structure of a complex analytic variety. This implies that we can integrate differential forms over these varieties (cf. [6], Chap. 0). With every divisor class $\mathcal{M}=$ $\mathcal{O}(\mathbf{d}) \in \operatorname{Pic}(\mathbf{n})$ we associate a $(1,1)$-differential form $c_{1}(\mathcal{M})$, called its $C h e r n$ form:

- if $m=1, \mathcal{M}=\mathcal{O}(1), \mathbf{n}=(n)$, then

$$
c_{1}(\mathcal{M}):=\omega_{n}=\frac{\sqrt{1}}{2 \pi} \partial \bar{\partial} \log \left(\left|Z_{0}\right|^{2}+\ldots+\left|Z_{n}\right|^{2}\right)
$$

is the $(1,1)$-form associated with the Fubini-Study metric on $\mathbb{P}^{n}(\mathbb{C})$, where $Z_{0}, \ldots, Z_{n}$ are the homogeneous coordinates on $\mathbb{P}^{n}$, cf. [6], p. 30 for an explicit formula;

- if $m \geq 1, \mathbf{d}=\left(d_{1}, \ldots, d_{m}\right)$, then

$$
c_{1}(\mathcal{M})=d_{1} \pi_{1}^{*} \omega_{n_{1}}+\ldots+d_{m} \pi_{m}^{*} \omega_{n_{m}},
$$

where $\pi_{h}$ is the projection $\mathbb{P}^{\mathbf{n}} \rightarrow \mathbb{P}^{n_{h}}$ and $\pi_{h}^{*} \omega_{n_{h}}$ is the pull back of $\omega_{n_{h}}$ from $\mathbb{P}^{n_{h}}$ to $\mathbb{P}^{\mathbf{n}}$ (i.e. $\pi_{h}^{*} \omega_{n_{h}}$ is defined by precisely the same formula as $\omega_{n_{h}}$ in terms of the homogeneous coordinates of $\mathbb{P}^{n_{h}}$ but it is considered as a differential form on $\mathbb{P}^{\mathbf{n}}$ ).

$(t, t)$-forms can be integrated over $t$-dimensional subvarieties of $\mathbb{P}^{\mathbf{n}}(\mathbb{C})$. For a cycle $Z=\sum n_{V} V \in \mathcal{Z}_{t}\left(\mathbb{P}^{\mathbf{n}}(\mathbb{C})\right)$ and a $(t, t)$-form $\varrho$ on $\mathbb{P}^{\mathbf{n}}(\mathbb{C})$, we set $\int_{Z} \varrho:=\sum n_{V} \int_{V} \varrho$. By Wirtinger's theorem (cf. [6], p. 171 or [7], Prop. 3.6), we have for $Z \in \mathcal{Z}_{t}\left(\mathbb{P}^{\mathbf{n}}(\mathbb{C})\right)$ and $\mathcal{M}_{1}, \ldots, \mathcal{M}_{t} \in \operatorname{Pic}(\mathbf{n})$,

$$
\int_{Z} c_{1}\left(\mathcal{M}_{1}\right) \wedge \ldots \wedge c_{1}\left(\mathcal{M}_{t}\right)=\left(Z \cdot \mathcal{M}_{1} \ldots \mathcal{M}_{t}\right) \text {. }
$$

The form $\omega_{n}$ is positive on $\mathbb{P}^{n}$ (cf. [6], p. 31). This implies that if $Z \in$ $\mathcal{Z}_{t}\left(\mathbb{P}^{\mathbf{n}}(\mathbb{C})\right)$ is effective, if $\mathcal{M}_{1}, \ldots, \mathcal{M}_{t} \in \operatorname{Pic}^{+}(\mathbf{n})$, and if $f$ is a real function which is non-negative everywhere on the components of $Z$, then

$$
\int_{Z} f \cdot c_{1}\left(\mathcal{M}_{1}\right) \wedge \ldots \wedge c_{1}\left(\mathcal{M}_{t}\right) \geq 0
$$

If $Z=\sum n_{P} P$ is a zero-dimensional cycle in $\mathbb{P}^{\mathbf{n}}(\mathbb{C})$ and $f$ is a function on $\mathbb{P}^{\mathbf{n}}(\mathbb{C})$ then we write $\int_{Z} f$ for $\sum n_{P} f(P)$. For $f \in \Gamma_{\mathbb{C}}^{\mathbf{n}}(\mathbf{d})$ we define a function $\|f\|$ on $\mathbb{P}^{\mathbf{n}}(\mathbb{C})$ as follows: let $\mathbf{Z}_{h}=\left(Z_{h 0}, \ldots, Z_{h, n_{h}}\right)$ be the complex homogeneous coordinates in $\mathbb{P}^{n_{h}}(\mathbb{C}), \mathbf{Z}=\left(\mathbf{Z}_{1}, \ldots, \mathbf{Z}_{m}\right),\left\|\mathbf{Z}_{h}\right\|=\left(\sum_{j=0}^{n_{h}}\left|Z_{h j}\right|^{2}\right)^{1 / 2}$; then put

where $\left(d_{1}, \ldots, d_{m}\right)=\mathbf{d}$.

$$
\|f\|(\mathbf{Z})=\frac{|f(\mathbf{Z})|}{\left\|\mathbf{Z}_{1}\right\|^{d_{1}} \cdots\left\|\mathbf{Z}_{m}\right\|^{d_{m}}},
$$


Let $Z$ be a subvariety of $\mathbb{P}^{\mathbf{n}}(\overline{\mathbb{Q}})$, defined over an algebraic number field $K$. Suppose that $f \in \Gamma^{\mathbf{n}}$ has its coefficients in $O_{K}$ and does not vanish identically on $Z$. Let $\mathcal{M}_{1}, \ldots, \mathcal{M}_{t} \in \operatorname{Pic}(\mathbf{n})$. Define, for each embedding $\sigma: K \hookrightarrow \mathbb{C}$,

$$
\begin{aligned}
\kappa_{\sigma} & =\kappa_{\sigma}\left(Z, f, \mathcal{M}_{1}, \ldots, \mathcal{M}_{t}\right) \\
& =-\frac{1}{[K: \mathbb{Q}]} \int_{Z \times_{\sigma} \mathbb{C}} \log \|\sigma(f)\| c_{1}\left(\mathcal{M}_{1}\right) \wedge \ldots \wedge c_{1}\left(\mathcal{M}_{t}\right),
\end{aligned}
$$

where $Z \times_{\sigma} \mathbb{C}=\left\{P \in \mathbb{P}^{\mathbf{n}}(\mathbb{C}): \sigma(g)(P)=0\right.$ for every $g \in K[\mathbf{X}]$ vanishing identically on $Z\}$.

Now let $\wp$ be a non-zero prime ideal of $O_{K}$. Let $I=\left\{f \in O_{K}[\mathbf{X}]\right.$ : $f(P)=0$ for all $P \in Z\}$. This is an essential prime ideal of $O_{K}[\mathbf{X}]$ with $I \cap O_{K}=0$. Let $J_{1, \wp}, \ldots, J_{g, \wp}$ be the minimal n-prime ideals of $O_{K}[\mathbf{X}]$ containing $I+\wp O_{K}[\mathbf{X}]$. Then

$$
W_{i \wp}:=\left\{\bar{P} \in \mathbb{P}^{\mathbf{n}}\left(\overline{\mathbb{F}}_{\wp}\right): \bar{g}(\bar{P})=0 \text { for } \bar{g} \in J_{i \wp} / \wp O_{K}[\mathbf{X}]\right\}
$$

is an $\mathbb{F}_{\wp}$-subvariety of $\mathbb{P}^{\mathbf{n}}\left(\overline{\mathbb{F}}_{\wp}\right)$ for $i=1, \ldots, g ; W_{1 \wp}, \ldots, W_{g \wp}$ may be considered as the irreducible components of the reduction of $Z \bmod \wp$. Define the local ring

$$
\mathcal{O}_{W_{i \wp}}=\left\{h / g: h, g \in \Gamma_{O_{K}}^{\mathbf{n}}(\mathbf{d}) \text { for some } \mathbf{d} \in\left(\mathbb{Z}_{\geq 0}\right)^{m}, g \notin J_{i_{\wp}}\right\},
$$

put $\operatorname{ord}_{W_{i_{\wp}}}(f \mid Z):=l_{\mathcal{O}_{W_{i \wp}}}\left(\mathcal{O}_{W_{i \wp}} /(I+(f)) \mathcal{O}_{W_{i_{\wp}}}\right)$ (which is finite since $I+(f)$ is a primary ideal for the maximal ideal of $\left.\mathcal{O}_{W_{i \wp}}\right)$, and define the $\wp$-divisor of $f$ restricted to $Z$,

$$
\operatorname{div}_{\wp}(f \mid Z)=\sum_{i=1}^{g} \operatorname{ord}_{W_{i \wp}}(f \mid Z) W_{i \wp} .
$$

For all but finitely many $\wp$ we have $\operatorname{div}_{\wp}(f \mid Z)=0$. Now put

$$
\kappa_{\wp}=\kappa_{\wp}\left(Z, f, \mathcal{M}_{1}, \ldots, \mathcal{M}_{t}\right)=\frac{\log N_{\wp}}{[K: \mathbb{Q}]}\left(\operatorname{div}_{\wp}(f \mid Z) \cdot \mathcal{M}_{1} \ldots \mathcal{M}_{t}\right),
$$

where the latter intersection number is for $\mathbb{F}_{\wp^{-}}$cycles. Finally, put

$$
\kappa_{K}\left(Z, f, \mathcal{M}_{1}, \ldots, \mathcal{M}_{t}\right):=\sum_{\sigma} \kappa_{\sigma}+\sum_{\wp} \kappa_{\wp},
$$

where the sums are over all embeddings $\sigma: K \hookrightarrow \mathbb{C}$ and all non-zero prime ideals $\wp$ of $O_{K}$. By linearity we define $\kappa_{\sigma}, \kappa_{\wp}, \kappa_{K}$ for cycles in $\mathcal{Z}_{t}$ with components defined over $K$.

The next result is due to Faltings [1]; implicitly, it implies that $\kappa_{K}$ is independent of $K$. 
Lemma 6. There are unique functions $h: \mathcal{Z}_{t}\left(\mathbb{P}^{\mathbf{n}}(\overline{\mathbb{Q}})\right) \times \operatorname{Pic}(\mathbf{n})^{t+1} \rightarrow \mathbb{R}$ for $t=0, \ldots, M$ (heights) with the following properties:

(i) $h\left(Z, \mathcal{M}_{0}, \ldots, \mathcal{M}_{t}\right)$ is additive in $Z, \mathcal{M}_{0}, \ldots, \mathcal{M}_{t}$ and invariant under permutations of $\mathcal{M}_{0}, \ldots, \mathcal{M}_{t}$;

(ii) for $Z \in \mathcal{Z}_{t}\left(\mathbb{P}^{\mathbf{n}}(\overline{\mathbb{Q}})\right), \mathcal{M}_{0} \in \operatorname{Pic}^{+}(\mathbf{n}), \mathcal{M}_{1}, \ldots, \mathcal{M}_{t} \in \operatorname{Pic}(\mathbf{n})$, choose any number field $K$ over which all components of $Z$ are defined, and choose any $f \in \Gamma\left(\mathcal{M}_{0}\right)$ with coefficients in $O_{K}$ such that $f$ does not identically vanish on $Z$. Then

$$
\begin{aligned}
h\left(Z, \mathcal{M}_{0}, \ldots, \mathcal{M}_{t}\right) & \\
& =h\left(\operatorname{div}(f \mid Z), \mathcal{M}_{1}, \ldots, \mathcal{M}_{t}\right)+\kappa_{K}\left(Z, f, \mathcal{M}_{1}, \ldots, \mathcal{M}_{t}\right) .
\end{aligned}
$$

R e m a r k. (3.5) holds true also for $t=0$, by agreeing that then $\operatorname{div}(f \mid Z)$ $=0$.

Proof of Lemma 6 . Put $R:=O_{K}$ and $\mathbb{P}_{R}^{\mathbf{n}}=\mathbb{P}_{R}^{n_{1}} \times_{\operatorname{Spec} R} \ldots \times_{\operatorname{Spec} R}$ $\mathbb{P}_{R}^{n_{m}}$. A subvariety of $\mathbb{P}_{R}^{\mathbf{n}}$ is by definition an integral closed subscheme of $\mathbb{P}_{R}^{\mathbf{n}}$ and a cycle in $\mathbb{P}_{R}^{\mathbf{n}}$ is a finite formal linear combination with integer coefficients of subvarieties of $\mathbb{P}_{R}^{\mathrm{n}}$. In [1], Faltings defined a logarithmic height for cycles in $\mathbb{P}_{R}^{\mathbf{n}}$ by means of the arithmetic intersection theory on $\mathbb{P}_{R}^{\mathbf{n}}$ developed by Gillet and Soulé [5], and he gave a sketchy proof of the analogue of our Lemma 6 for cycles in $\mathbb{P}_{R}^{\mathbf{n}}$. A more detailed proof of this analogue was given by Gubler [7], Props. 4.3, 5.3.

It is straightforward to translate Gubler's results into Lemma 6 by going through the definition of a scheme. Similar to [8], Ex. 3.12 on p. 92, 5.16 on pp. 119-120 and Ex. 5.10 on p. 125, there is a one-to-one correspondence $I \leftrightarrow V(I)$ between essential $\mathbf{n}$-prime ideals of $R[\mathbf{X}]$ and subvarieties of $\mathbb{P}_{R}^{\mathbf{n}}$, such that $V(I)$ is a subvariety of $V(J) \Leftrightarrow I \supseteq J$. Further, for subvarieties $V(I)$ of $\mathbb{P}_{R}^{\text {n }}$ we have either $I \cap R=(0)$, in which case $V(I)$ is flat (over Spec $R$ ) (cf. [8], p. 257, Prop. 9.7), or $I \cap R$ is a non-zero prime ideal $\wp$ of $R$, in which case $V(I)$ maps to $\wp$ (under $V(I) \rightarrow \operatorname{Spec} R$ ).

Now let $Z$ be a subvariety of $\mathbb{P}^{\mathbf{n}}$ defined over $K$, and let $I=\{f \in R[\mathbf{X}]$ : $f(P)=0$ for $P \in Z\}$. Then $\widetilde{Z}:=V(I)$ is a flat subvariety of $\mathbb{P}_{R}^{\mathbf{n}}$. Now the height $h(Z, \cdot)$ defined in Lemma 6 is equal to the height $h(\widetilde{Z}, \cdot)$ defined by Gubler (and $1 /[K: \mathbb{Q}]$ times the height defined by Faltings). Faltings [1] and Gubler [7], Proposition 4.3, have a similar recurrence relation as (3.5) for the height of flat subvarieties $\widetilde{Z}$ of $\mathbb{P}_{R}^{\mathbf{n}}$, with instead of $\kappa_{K}$ only the sum of infinite components $\kappa_{\sigma}$. The $\operatorname{divisor} \operatorname{div}(f \mid \widetilde{Z})$ might have also non-flat components and the terms $\kappa_{\wp}$ in (3.5) are precisely the contributions of the heights of these non-flat components. By Proposition 5.3 of [7], the Faltings height for subvarieties of $\mathbb{P}_{R}^{\mathbf{n}}$ is invariant under base extensions from $R$ to the ring of integers of any finite extension of $K$. This implies that in Lemma 6 , the height does not depend on the choice of the field $K$. 
For $Z \in \mathcal{Z}_{t}\left(\mathbb{P}^{\mathbf{n}}\right)$ and non-negative integers $e_{1}, \ldots, e_{s}$ with $e_{1}+\ldots+$ $e_{s}=t+1$, we write $h\left(Z, \mathcal{N}_{1}^{e_{1}} \ldots \mathcal{N}_{s}^{e_{s}}\right)$ for $h(Z, \underbrace{\mathcal{N}_{1}, \ldots, \mathcal{N}_{1}}_{e_{1} \text { times }}, \ldots, \underbrace{\mathcal{N}_{s}, \ldots, \mathcal{N}_{s}}_{e_{s} \text { times }})$.

Further, for $Z \in \mathcal{Z}_{t}\left(\mathbb{P}^{n}\right)$ we put $h(Z):=h\left(Z, \mathcal{O}(1)^{t+1}\right)$. We write again $\mathbb{P}^{\mathbf{n}}$ for $\mathbb{P}^{\mathbf{n}}(\overline{\mathbb{Q}})$.

Lemma 7. (i) For $P \in \mathbb{P}^{n}$ we have $h(P)=\log H(P)$.

(ii) $h\left(\mathbb{P}^{n}\right)=\frac{1}{2} \sum_{j=1}^{n} \sum_{l=1}^{j} 1 / l$.

(iii) If $Z \in \mathcal{Z}_{t}\left(\mathbb{P}^{\mathbf{n}}\right)$ is effective and $\mathcal{M}_{0}, \ldots, \mathcal{M}_{t} \in \operatorname{Pic}^{+}(\mathbf{n})$, then $h\left(Z, \mathcal{M}_{0}, \ldots, \mathcal{M}_{t}\right) \geq 0$.

(iv) Let $Z, \mathcal{M}_{0}, \ldots, \mathcal{M}_{t}$ be as in (iii) and $f \in \Gamma\left(\mathcal{M}_{0}\right)$ such that $f$ does not identically vanish on $Z$. Then

$h\left(\operatorname{div}(f \mid Z), \mathcal{M}_{1}, \ldots, \mathcal{M}_{t}\right) \leq h\left(Z, \mathcal{M}_{0}, \ldots, \mathcal{M}_{t}\right)+\log H(f) \cdot\left(Z \cdot \mathcal{M}_{1} \ldots \mathcal{M}_{t}\right)$.

Proof. (i) In a sufficiently large number field $K$ we can choose the coordinates $\mathbf{x}=\left(x_{0}, \ldots, x_{n}\right)$ of $P$ such that $x_{0}, \ldots, x_{n} \in O_{K}$ and the ideal generated by these coordinates is (1). Then there are $\alpha_{0}, \ldots, \alpha_{n} \in O_{K}$ with $\alpha_{0} x_{0}+\ldots+\alpha_{n} x_{n}=1$. Take $f(\mathbf{X})=\alpha_{0} X_{0}+\ldots+\alpha_{n} X_{n}$. Then $f$ does not vanish at $P, \operatorname{div}(f \mid \mathcal{M})=0, \kappa_{\wp}(P, f)=0$ for each prime ideal $\wp \neq(0)$ of $O_{K}$ and

$$
\kappa_{\sigma}(P, f)=-\frac{1}{[K: \mathbb{Q}]} \log \frac{|\sigma(f)(\mathbf{x})|}{\left(\sum_{i=0}^{n}\left|\sigma\left(x_{i}\right)\right|^{2}\right)^{1 / 2}}=\log \left\{\left(\sum_{i=0}^{n}\left|\sigma\left(x_{i}\right)\right|^{2}\right)^{1 / 2[K: \mathbb{Q}]}\right\} .
$$

Hence

$$
h(P)=h(\operatorname{div}(f \mid P))=\frac{1}{[K: \mathbb{Q}]} \sum_{\sigma} \kappa_{\sigma}(P, f)=\log H(P) .
$$

(ii) (cf. [7], Prop. 4.4). This can be proved by induction on $n$. Take $f=X_{0}$. Then $h\left(\mathbb{P}^{n}\right)=h\left(\operatorname{div}\left(f \mid \mathbb{P}^{n}\right)\right)+\kappa=h(V)+\kappa$, where $V=\left\{X_{0}=0\right\}$ and

$$
\kappa=-\int_{\mathbb{P}^{n}(\mathbb{C})} \log \frac{\left|z_{0}\right|}{\left(\left|z_{0}\right|^{2}+\ldots+\left|z_{n}\right|^{2}\right)^{1 / 2}} \cdot \omega_{n} .
$$

By the induction hypothesis, $h(V)=h\left(\mathbb{P}^{n-1}\right)=\frac{1}{2} \sum_{j=1}^{n-1} \sum_{l=1}^{j} 1 / l$ and, by a straightforward but elaborate integration, $\kappa=\frac{1}{2} \sum_{l=1}^{n} 1 / l$.

(iv) We assume that $Z$ is a subvariety of $\mathbb{P}^{\mathbf{n}}$, which is no restriction. Choose a number field $K$ such that $Z$ and the components of $\operatorname{div}(f \mid Z)$ are defined over $K$ and the coefficients of $f$ belong to $K$. By enlarging $K$ if need be, we may assume that the ideal $\mathfrak{a}$ generated by the coefficients of $f$ is principal, $\mathfrak{a}=(\lambda)$, say. Since $\operatorname{div}(f \mid Z)$ and $H(f)$ do not change when $f$ is replaced by $\lambda^{-1} f$, we may assume that $\mathfrak{a}=(1)$ and shall do so in the sequel. Suppose $\mathcal{M}_{0}=\mathcal{O}(\mathbf{d})$, with $\mathbf{d}=\left(d_{1}, \ldots, d_{m}\right) \in\left(\mathbb{Z}_{\geq_{0}}\right)^{m}$. Let $\mathcal{J}$ be the set of 
tuples of non-negative integers $\mathbf{i}=\left(i_{h j}: h=1, \ldots, m, j=0, \ldots, n_{h}\right)$ with $\sum_{j=0}^{n_{h}} i_{h j}=d_{h}$ for $h=1, \ldots, m$. Then

$$
f=\sum_{\mathbf{i} \in \mathcal{J}} a(\mathbf{i}) \prod_{h=1}^{m} \prod_{j=0}^{n_{h}} X_{h j}^{i_{h j}} \quad \text { with } a(\mathbf{i}) \in K .
$$

Let $\sigma$ be an embedding $K \hookrightarrow \mathbb{C}$ and $A_{\sigma}:=\left(\sum_{\mathbf{i} \in \mathcal{J}}|\sigma(a(\mathbf{i}))|^{2}\right)^{1 / 2}$. By Schwarz' inequality we have for $\mathbf{z}=\left(\mathbf{z}_{1}, \ldots, \mathbf{z}_{m}\right)$ with $\mathbf{z}_{h}=\left(z_{h 0}, \ldots, z_{h, n_{h}}\right) \in$ $\mathbb{C}^{n_{h}+1}$ for $h=1, \ldots, m$ that

$$
|\sigma(f)(\mathbf{z})|=\left|\sum_{i \in \mathcal{J}} \sigma(a(\mathbf{i})) \prod_{h=1}^{m} \prod_{j=0}^{n_{m}} z_{h_{j}}^{i_{h_{j}}}\right| \leq A_{\sigma}\left\|\mathbf{z}_{1}\right\|^{d_{1}} \ldots\left\|\mathbf{z}_{m}\right\|^{d_{m}} .
$$

Hence $\|\sigma(f)\| \leq A_{\sigma}$. Together with (3.2), (3.1) this implies that

$$
\begin{aligned}
\kappa_{\sigma} & \geq-\frac{1}{[K: \mathbb{Q}]} \log A_{\sigma} \int_{Z} c_{1}\left(\mathcal{M}_{1}\right) \wedge \ldots \wedge c_{1}\left(\mathcal{M}_{t}\right) \\
& \geq-\frac{1}{[K: \mathbb{Q}]} \log A_{\sigma}\left(Z \cdot \mathcal{M}_{1} \ldots \mathcal{M}_{t}\right),
\end{aligned}
$$

where $\kappa_{\sigma}=\kappa_{\sigma}\left(Z, f, \mathcal{M}_{1}, \ldots, \mathcal{M}_{t}\right)$. Further, for every non-zero prime ideal $\wp$ of $O_{K}$ we have $\kappa_{\wp}=\kappa_{\wp}\left(Z, f, \mathcal{M}_{1}, \ldots, \mathcal{M}_{t}\right) \geq 0$. Since $\mathfrak{a}=(1)$ we have $H(f)=\left(\prod_{\sigma} A_{\sigma}\right)^{1 /[K: \mathbb{Q}]}$. It follows that

$$
\begin{aligned}
h\left(\operatorname{div}(f \mid Z), \mathcal{M}_{1}, \ldots, \mathcal{M}_{t}\right) & \\
& =h\left(Z, \mathcal{M}_{0}, \ldots, \mathcal{M}_{t}\right)-\sum_{\sigma} \kappa_{\sigma}-\sum_{\wp} \kappa_{\wp} \\
& \leq h\left(Z, \mathcal{M}_{0}, \ldots, \mathcal{M}_{t}\right)+(\log H(f))\left(Z \cdot \mathcal{M}_{1} \ldots \mathcal{M}_{t}\right) .
\end{aligned}
$$

(iii) Apply (iv) with $f$ a monomial. Then $\log H(f)=0$; and therefore

$$
h\left(\operatorname{div}(f \mid Z), \mathcal{M}_{1}, \ldots, \mathcal{M}_{t}\right) \leq h\left(\mathcal{M}_{0}, \ldots, \mathcal{M}_{t}\right) .
$$

Now (iii) follows easily by induction on $t$.

Lemma 8. (i) Let $Z=Z_{1} \times \ldots \times Z_{m}$ where $Z_{h} \in \mathcal{Z}_{\delta_{h}}\left(\mathbb{P}^{n_{h}}\right)$ for $h=$ $1, \ldots, m$ and put $\delta=\delta_{1}+\ldots+\delta_{m}$. Further, let $e_{1}, \ldots, e_{m}$ be non-negative integers with $e_{1}+\ldots+e_{m}=\delta+1$. Then

$$
h\left(Z, \mathcal{L}_{1}^{e_{1}} \ldots \mathcal{L}_{m}^{e_{m}}\right)=h\left(Z_{h}\right) \prod_{j \neq h} \operatorname{deg} Z_{j}
$$

if for some $h \in\{1, \ldots, m\}$ we have $\left(e_{1}, \ldots, e_{m}\right)=\left(\delta_{1}, \ldots, \delta_{h-1}, \delta_{h}+1, \ldots\right.$ $\left.\ldots, \delta_{m}\right)$, and $h\left(Z, \mathcal{L}_{1}^{e_{1}} \ldots \mathcal{L}_{m}^{e_{m}}\right)=0$ otherwise. 
(ii)

$$
\begin{aligned}
h\left(Z, \mathcal{L}^{\delta+1}\right)= & d_{1}^{\delta_{1}} \ldots d_{m}^{\delta_{m}} \sum_{h=1}^{m} \frac{(\delta+1) !}{\delta_{1} ! \ldots\left(\delta_{h}+1\right) ! \ldots \delta_{m} !} \\
& \times\left(d_{h} h\left(Z_{h}\right) \prod_{j \neq h} \operatorname{deg} Z_{j}\right) .
\end{aligned}
$$

Proof. (i) This was stated without proof by Faltings [1]. We assume that $e_{1}-\delta_{1} \geq \ldots \geq e_{m}-\delta_{m}$ and that $Z_{h}$ is a $\delta_{h}$-dimensional subvariety of $\mathbb{P}^{n_{h}}$ for $h=1, \ldots, m$, which are no restrictions. For convenience of notation, put $c=1$ if $\left(e_{1}-\delta_{1}, \ldots, e_{m}-\delta_{m}\right)=(1,0, \ldots, 0)$ and $c=0$ otherwise.

We proceed by induction on $\delta_{1}$. Note that $e_{1}-\delta_{1} \geq 1$; hence $e_{1} \geq 1$. Choose a number field $K$ and a linear form $f \in O_{K}\left[\mathbf{X}_{1}\right]$ such that $f$ does not vanish identically on $Z_{1}$, and such that $Z_{1}, \ldots, Z_{m}$ and the components of $\operatorname{div}\left(f \mid Z_{1}\right), \operatorname{div}\left(\pi_{1}^{*} f \mid Z\right)$ are defined over $K$. Consider the quantities

$$
\begin{aligned}
U & :=h\left(Z, \mathcal{L}_{1}^{e_{1}} \ldots \mathcal{L}_{m}^{e_{m}}\right)-\operatorname{ch}\left(Z_{1}\right) \prod_{h=2}^{m} \operatorname{deg} Z_{h}, \\
u & :=h\left(\operatorname{div}\left(\pi_{1}^{*} f \mid Z\right), \mathcal{L}_{1}^{e_{1}-1} \mathcal{L}_{2}^{e_{2}} \ldots \mathcal{L}_{m}^{e_{m}}\right)-\operatorname{ch}\left(\operatorname{div}\left(f \mid Z_{1}\right)\right) \prod_{h=2}^{m} \operatorname{deg} Z_{h} .
\end{aligned}
$$

If $\delta_{1}=0$ then $\operatorname{div}\left(\pi_{1}^{*} f \mid Z\right)=0, \operatorname{div}\left(f \mid Z_{1}\right)=0$ hence $u=0$; if $\delta_{1}>0$ then also $u=0$ by the induction hypothesis. We have to show that $U=0$.

By Lemma 6(ii) we have

$$
U=U-u=\sum_{\sigma} \lambda_{\sigma}+\sum_{\wp} \lambda_{\wp},
$$

where

$$
\lambda_{v}=\kappa_{v}\left(Z, \pi_{1}^{*} f, \mathcal{L}_{1}^{e_{1}-1} \ldots \mathcal{L}_{m}^{e_{m}}\right)-c \operatorname{deg} Z_{2} \ldots \operatorname{deg} Z_{m} \kappa_{v}\left(Z_{1}, f, \mathcal{O}(1)^{e_{1}-1}\right)
$$

for $v \in\{\sigma\} \cup\{\wp\}$, where $\sigma$ stands for the embeddings of $K$ in $\mathbb{C}$ and $\wp$ for the non-zero prime ideals of $O_{K}$. If $\left(e_{1}-\delta_{1}, \ldots, e_{m}-\delta_{m}\right)=(1,0, \ldots, 0)$, then by (3.1) we have for each embedding $\sigma: K \hookrightarrow \mathbb{C}$,

$$
\begin{aligned}
\kappa_{\sigma}\left(Z, \pi_{1}^{*} f, \mathcal{L}_{1}^{e_{1}-1} \ldots \mathcal{L}_{m}^{e_{m}}\right) \\
=-\frac{1}{[K: \mathbb{Q}]} \int_{Z \times_{\sigma} \mathbb{C}} \log \left\|\sigma\left(\pi_{1}^{*} f\right)\right\| c_{1}\left(\mathcal{L}_{1}\right)^{e_{1}-1} \wedge \ldots \wedge c_{1}\left(\mathcal{L}_{m}\right)^{e_{m}} \\
\quad=-\frac{1}{[K: \mathbb{Q}]} \int_{Z_{1} \times_{\sigma} \mathbb{C}} \log \|\sigma(f)\| \omega_{n_{1}}^{e_{1}-1} \cdot \prod_{h=2}^{m} \int_{Z_{h} \times_{\sigma} \mathbb{C}} \omega_{n_{h}}^{e_{h}} \\
=\kappa_{\sigma}\left(Z_{1}, f_{1}, \mathcal{O}(1)^{e_{1}-1}\right) \operatorname{deg} Z_{2} \ldots \operatorname{deg} Z_{m} .
\end{aligned}
$$


If $\left(e_{1}-\delta_{1}, \ldots, e_{m}-\delta_{m}\right) \neq(1,0, \ldots, 0)$ then $\kappa_{\sigma}\left(Z, \pi_{1}^{*} f, \mathcal{L}_{1}^{e_{1}-1} \ldots \mathcal{L}_{m}^{e_{m}}\right)=0$; namely in that case either $e_{1}-1>\delta_{1}$ or $e_{h}>\delta_{h}$ for some $h \geq 2$, which implies that the restriction of the differential form $c_{1}\left(\mathcal{L}_{1}\right)^{e_{1}-1} \wedge \ldots \wedge c_{1}\left(\mathcal{L}_{m}\right)^{e_{m}}$ to $Z_{h}$ has degree larger than $2 \operatorname{dim} Z_{h}$, which is the dimension of $Z_{h}$ over $\mathbb{R}$. It follows that in both cases,

$$
\lambda_{\sigma}=0 \quad \text { for each embedding } \sigma: K \hookrightarrow \mathbb{C} .
$$

Let $p$ be any prime number and for each prime ideal $\wp$ of $O_{K}$ dividing $p$, put $d_{\wp}:=\left[\mathbb{F}_{\wp}: \mathbb{F}_{p}\right]$. Then

$$
\sum_{\wp \mid p} \lambda_{\wp}=n_{p}(f) \log p
$$

where

$$
\begin{aligned}
n_{p}(f)=\frac{1}{[K: \mathbb{Q}]} \sum_{\wp \mid p} f_{\wp}\{ & \left(\operatorname{div}_{\wp}\left(\pi_{1}^{*} f \mid Z\right) \cdot \mathcal{L}_{1}^{e_{1}-1} \ldots \mathcal{L}_{m}^{e_{m}}\right) \\
& \left.-c \operatorname{deg} Z_{2} \ldots \operatorname{deg} Z_{m}\left(\operatorname{div}_{\wp}\left(f \mid Z_{1}\right) \cdot \mathcal{O}(1)^{e_{1}-1}\right)\right\} .
\end{aligned}
$$

By (3.7), (3.8) we have

$$
U=\sum_{p} n_{p}(f) \log p
$$

hence the right-hand side of (3.9) is independent of the choice of $f$ and $K$. But by the unique prime decomposition in $\mathbb{Z}$ the numbers $\log p$ ( $p$ prime) are $\mathbb{Q}$-linearly independent; therefore the rational numbers $n_{p}(f)$ are independent of the choice of $f$ and $K$.

We show that for every prime number $p$ we can choose $f$ with $n_{p}(f)=0$. Let $I=\left\{g \in O_{K}[\mathbf{X}]: g\right.$ vanishes identically on $\left.Z\right\}$ and $J_{1}, \ldots, J_{g}$ the minimal n-prime ideals of $O_{K}[\mathbf{X}]$ containing at least one of the ideals $I+$ $\wp O_{K}[\mathbf{X}]$ with $\wp \mid p$. Let $I^{\prime}=\left\{g^{\prime}\right.$ vanishes identically on $\left.Z_{1}\right\}$, and $J_{1}^{\prime}, \ldots, J_{g^{\prime}}^{\prime}$ the minimal homogeneous prime ideals of $O_{K}\left[\mathbf{X}_{1}\right]$ containing at least one of the ideals $I^{\prime}+\wp O_{K}\left[\mathbf{X}_{1}\right]$ with $\wp \mid p$. Choose a linear form $f \in O_{K}\left[\mathbf{X}_{1}\right]$ with $f \notin \pi_{1}^{*-1}\left(J_{1}\right) \cup \ldots \cup \pi_{1}^{*-1}\left(J_{g}\right) \cup J_{1}^{\prime} \cup \ldots \cup J_{g^{\prime}}^{\prime}$. Such an $f$ exists since each of the ideals in the union is a homogeneous prime ideal not containing $\left(X_{10}, \ldots, X_{1, n_{1}}\right)$. Thus, $\operatorname{div}_{\wp}\left(\pi_{1}^{*} f \mid Z\right)=0, \operatorname{div}_{\wp}\left(f \mid Z_{1}\right)=0$ for every $\wp \mid p$, which implies that $n_{p}(f)=0$. Now (3.9) implies that $U=0$. This completes the proof of (i).

(ii) By the additivity of the height and (i) we have

$$
\begin{aligned}
h\left(Z_{1}, \mathcal{L}^{\delta+1}\right) & =h\left(Z,\left(d_{1} \mathcal{L}_{1}+\ldots+d_{m} \mathcal{L}_{m}\right)^{\delta+1}\right) \\
& =\sum_{e_{1}+\ldots+e_{m}=\delta+1} \frac{(\delta+1) !}{e_{1} ! \ldots e_{m} !} d_{1}^{e_{1}} \ldots d_{m}^{e_{m}} h\left(Z, \mathcal{L}_{1}^{e_{1}} \ldots \mathcal{L}_{m}^{e_{m}}\right)
\end{aligned}
$$




$$
=\sum_{h=1}^{m} \frac{(\delta+1) !}{\delta_{1} ! \ldots\left(\delta_{h}+1\right) ! \ldots \delta_{m} !} d_{1}^{\delta_{1}} \ldots d_{h}^{\delta_{h}+1} \ldots d_{m}^{\delta_{m}}\left(h\left(Z_{h}\right) \prod_{j \neq h} \operatorname{deg} Z_{j}\right),
$$

which is (ii).

Finally, we need an analogue of Lemma 4 for heights. For a polynomial $f\left(X_{1}, \ldots, X_{r}\right)=\sum_{\mathbf{i}} a(\mathbf{i}) X_{1}^{i_{1}} \ldots X_{r}^{i_{r}}$ with coefficients in a number field $K$ and for each embedding $\sigma: K \hookrightarrow \mathbb{C}$, put

$$
H_{\sigma}(f)=\left(\sum_{\mathbf{i}}|\sigma(a(\mathbf{i}))|^{2}\right)^{1 / 2} .
$$

Lemma 9. Let $\mathbf{d}=\left(d_{1}, \ldots, d_{m}\right) \in\left(\mathbb{Z}_{\geq 0}\right)^{m}$ and $A$ a subset of $\Gamma_{\mathbb{Q}}^{\mathbf{n}}(\mathbf{d}) \backslash\{\mathbf{0}\}$ such that every polynomial $f \in A$ has algebraic integer coefficients in some number field $K$ and such that $H_{\sigma}(f) \leq H_{\sigma}$ for each embedding $\sigma: K \hookrightarrow \mathbb{C}$. Put $H:=\left(\prod_{\sigma} H_{\sigma}\right)^{1 /[K: \mathbb{Q}]}$. Further, let $Z_{1}, \ldots, Z_{r}$ be irreducible components of $X:=\left\{P \in \mathbb{P}^{\mathbf{n}}(\overline{\mathbb{Q}}): f(P)=0\right.$ for $\left.f \in A\right\}$ of codimension $t$. Then

$\sum_{i=1}^{r} m_{Z_{i}} h\left(Z_{i}, \mathcal{L}^{M-t+1}\right) \leq \frac{M !}{n_{1} ! \ldots n_{m} !} d_{1}^{n_{1}} \ldots d_{m}^{n_{m}}\left\{M^{2}\left(d_{1}+\ldots+d_{m}\right)+t \log H\right\}$.

Pro o f. Let $f_{1}, \ldots, f_{t}$ be polynomials satisfying (2.6) and Lemma 5 , and $C_{0}, \ldots, C_{t}$ the cycles defined by $(2.6)$; so $C_{0}=\mathbb{P}^{\mathbf{n}}$. From the definition of the height of a polynomial and the fact that the quantities $H_{\sigma}(f)$ satisfy the triangle inequality it follows that

$$
H\left(f_{i}\right) \leq C^{2} H \quad \text { for } i=1, \ldots, t, \quad \text { where } C=\frac{M !}{n_{1} ! \ldots n_{m} !} d_{1}^{n_{1}} \ldots d_{m}^{n_{m}} .
$$

By Lemma 7(iv) we have

$$
h\left(\mathbb{P}^{n}\right)=\frac{1}{2} \sum_{j=1}^{n} \sum_{l=1}^{j} \frac{1}{l} \leq \frac{1}{2}(n+\log n !) .
$$

Together with Lemma 8(ii) this implies

$$
\begin{aligned}
h\left(\mathbb{P}^{\mathbf{n}}, \mathcal{L}^{M+1}\right) & =d_{1}^{n_{1}} \ldots d_{m}^{n_{m}} \sum_{h=1}^{m} \frac{(M+1) !}{n_{1} ! \ldots\left(n_{h}+1\right) ! \ldots n_{m} !} d_{h} h\left(\mathbb{P}^{n_{h}}\right) \\
& \leq \frac{1}{2} C(M+1) \sum_{h=1}^{m} \frac{n_{h}+\log n_{h} !}{n_{h}+1} d_{h} \\
& \leq \frac{1}{4} C(M+1) \sum_{h=1}^{m} n_{h} d_{h} \leq \frac{1}{4} C M^{2}\left(d_{1}+\ldots+d_{m}\right) .
\end{aligned}
$$

By (2.8) we have $\operatorname{div}\left(f_{j} \mid C_{j-1}\right)=C_{j}+$ (effective cycle) for $j=1, \ldots, t$. By Lemma 1 (iv) we have $\left(C_{j} \cdot \mathcal{L}^{M-j}\right) \leq\left(C_{j-1} \cdot \mathcal{L}^{M-j+1}\right) \leq \ldots \leq\left(C_{0} \cdot \mathcal{L}^{M}\right)=C$ 
for $j=0, \ldots, t$. Further, by Lemma 7(iii), (iv) and (3.10),

$$
\begin{aligned}
h\left(C_{j}, \mathcal{L}^{M-j+1}\right) & \leq h\left(C_{j-1}, \mathcal{L}^{M-j+2}\right)+\left(\log C^{2} H\right)\left(C_{j-1} \mathcal{L}^{M-j+1}\right) \\
& \leq h\left(C_{j-1}, \mathcal{L}^{M-j+2}\right)+C \log \left(C^{2} H\right)
\end{aligned}
$$

for $j=1, \ldots, t$. Now from $C_{t}=\sum_{i=1}^{r} m_{Z_{i}} Z_{i}+$ (effective cycle), (3.12), (3.11), $C \leq \sum_{j_{1}+\ldots+j_{m}=M}\left(M ! /\left(j_{1} ! \ldots j_{m} !\right)\right) d_{1}^{j_{1}} \ldots d_{m}^{j_{m}}=\left(d_{1}+\ldots+d_{m}\right)^{M}$, $\log \left(d_{1}+\ldots+d_{m}\right) \leq(\log 3 / 3)\left(d_{1}+\ldots+d_{m}\right)$ and $t \leq M$ it follows that

$$
\begin{aligned}
\sum_{i=1}^{r} m_{Z_{i}} h\left(Z_{i}, \mathcal{L}^{M-t+1}\right) \\
\leq h\left(C_{t}, \mathcal{L}^{M-t+1}\right) \leq h\left(\mathbb{P}^{\mathbf{n}}, \mathcal{L}^{M+1}\right)+C t \log \left(C^{2} H\right) \\
\leq C\left\{\frac{1}{4} M^{2}\left(d_{1}+\ldots+d_{m}\right)+2 t \log C+t \log H\right\} \\
\leq C\left\{\frac{1}{4} M^{2}\left(d_{1}+\ldots+d_{m}\right)+2 t M \log \left(d_{1}+\ldots+d_{m}\right)+t \log H\right\} \\
\leq C\left\{\left(\frac{1}{4}+2 \frac{\log 3}{3}\right) M^{2}\left(d_{1}+\ldots+d_{m}\right)+t \log H\right\} \\
\leq C\left\{M^{2}\left(d_{1}+\ldots+d_{m}\right)+t \log H\right\}
\end{aligned}
$$

which is Lemma 9.

4. Proof of Theorems 1 and 2. We use the notation of Theorem $1: k$ is an algebraically closed field of characteristic $0, m$ an integer $\geq 2$, $\mathbf{n}=\left(n_{1}, \ldots, n_{m}\right)$ and $\mathbf{d}=\left(d_{1}, \ldots, d_{m}\right)$ tuples of positive integers and $\sigma, \varepsilon$ reals with $\sigma \geq 0,0<\varepsilon \leq 1$ and

$$
\frac{d_{h}}{d_{h+1}} \geq\left(\frac{m M}{\varepsilon}\right)^{M} \quad \text { for } h=1, \ldots, m-1,
$$

where $M:=n_{1}+\ldots+n_{m}$. We write $\mathbb{P}^{\mathbf{n}}$ for $\mathbb{P}^{\mathbf{n}}(k)$. Further, $F$ is a polynomial from $\Gamma_{k}^{\mathbf{n}}(\mathbf{d}) \backslash\{0\}$, and $Z$ is an irreducible component of both $Z_{\sigma}(F, \mathbf{d})$ and $Z_{\sigma+\varepsilon}(F, \mathbf{d})$. Let $A$ be the set of polynomials

$$
\prod_{h=1}^{m} \prod_{j=0}^{n_{h}} X_{h j}^{c_{h j}}\left(\prod_{h=1}^{m} \prod_{j=0}^{n_{h}} \frac{1}{i_{h j} !} \cdot \frac{\partial^{i_{h j}}}{\partial X_{h j}^{i_{h j}}} F\right)
$$

for all tuples of non-negative integers $\mathbf{i}=\left(i_{h j}: h=1, \ldots, m, j=0, \ldots, n_{h}\right)$, $\mathbf{c}=\left(c_{h j}: h=1, \ldots, m, j=0, \ldots, n_{h}\right)$ with

$$
(\mathbf{i} / \mathbf{d}) \leq \sigma, \quad \sum_{j=0}^{n_{h}}\left(c_{h j}+i_{h j}\right)=d_{h} \quad \text { for } h=1, \ldots, m
$$

and let $I$ be the ideal in $k[\mathbf{X}]$ generated by $A$. Note that $A \subset \Gamma_{k}^{\mathbf{n}}(\mathbf{d})$, and that $X:=Z_{\sigma}(F, \mathbf{d})=\left\{P \in \mathbb{P}^{\mathbf{n}}: f(P)=0\right.$ for $\left.P \in A\right\}$. 
Let $k_{0}$ be a subfield of $k$ containing the coefficients of $F$, and $k_{1}$ the smallest extension of $k_{0}$ over which $Z$ is defined. Thus, $Z=V(J)$ with $J=\left(f_{1}, \ldots, f_{u}\right)$, where $f_{1}, \ldots, f_{u} \in k_{1}[\mathbf{X}]$. Letting $\sigma_{1}=$ identity, $\sigma_{2}, \ldots, \sigma_{g}$ $\left(g=\left[k_{1}: k_{0}\right]\right)$ be the injective $k_{0}$-homomorphisms from $k_{1}$ into $k$, put $J^{(j)}:=\left(\sigma_{j}\left(f_{1}\right), \ldots, \sigma_{j}\left(f_{u}\right)\right), Z^{(j)}=V\left(J^{(j)}\right)$ for $j=1, \ldots, g$. Since $A \subset$ $k_{0}[\mathbf{X}], Z^{(1)}, \ldots, Z^{(g)}$ are irreducible components of $X$. Each $\sigma_{j}$ induces an isomorphism $\bar{\sigma}_{j}$ from $\mathcal{O}_{Z}$ to $\mathcal{O}_{Z^{(j)}}$ mapping the maximal ideal $M_{Z}$ to $M_{Z^{(j)}}$.

It follows that the fields $k\left(Z^{(j)}\right)$ and $k(Z)$ are isomorphic, whence $\operatorname{dim} Z^{(j)}=\operatorname{dim} Z$ for $j=1, \ldots, g$. Further, since $I$ is generated by polynomials from $k_{0}[\mathbf{X}], \bar{\sigma}_{j}$ induces an isomorphism from $\mathcal{O}_{Z} / I \mathcal{O}_{Z}$ to $\mathcal{O}_{Z^{(j)}} / I \mathcal{O}_{Z^{(j)}}$. Therefore,

$$
m_{Z^{(j)}}=m_{Z}
$$

(cf. Lemma 4). Let $s:=\operatorname{codim} Z$ and let $e_{1}, \ldots, e_{m}$ be non-negative integers with $e_{1}+\ldots+e_{m}=M-s$. Let $\mathcal{L}_{1}, \ldots, \mathcal{L}_{m}$ have the same meaning as in Section 2 , and put $\mathcal{L}:=d_{1} \mathcal{L}_{1}+\ldots+d_{m} \mathcal{L}_{m}$. By applying Lemma 1(iv) with polynomials from $k_{0}[\mathbf{X}]$, we infer that

$$
\left(Z^{(j)} \cdot \mathcal{L}_{1}^{e_{1}} \ldots \mathcal{L}_{m}^{e_{m}}\right)=\left(Z \cdot \mathcal{L}_{1}^{e_{1}} \ldots \mathcal{L}_{m}^{e_{m}}\right) \quad \text { for } j=1, \ldots, g .
$$

Together with (4.2) and Lemma 4, this implies that

$$
\left[k_{1}: k_{0}\right] m_{Z}\left(Z \cdot \mathcal{L}_{1}^{e_{1}} \ldots \mathcal{L}_{m}^{e_{m}}\right) \leq\left(\mathcal{L}_{1}^{e_{1}} \ldots \mathcal{L}_{m}^{e_{m}} \cdot \mathcal{L}^{s}\right)
$$

We shall estimate $m_{Z}$ from below, using differential operators similar to Wüstholz [20]. Here it will be crucial that $Z$ is also an irreducible component of $Z_{\sigma+\varepsilon}(F, \mathbf{d})$. If $Z$ is not a product variety then by Lemma 3 there are at least two tuples $\left(e_{1}, \ldots, e_{m}\right)$ for which $\left(Z \cdot \mathcal{L}_{1}^{e_{1}} \ldots \mathcal{L}_{m}^{e_{m}}\right)>0$. Using (1.1) and the lower bound for $m_{Z}$, we show that for some tuple $\left(e_{1}, \ldots, e_{m}\right)$, the left-hand side of (4.3) is larger than the right-hand side, thus arriving at a contradiction.

Lemma 10. For $i=1, \ldots, m$, let $p_{i}: \mathbb{P}^{\mathbf{n}} \rightarrow \mathbb{P}^{n_{i}} \times \ldots \times \mathbb{P}^{n_{m}}$ be the projection onto the last $m-i+1$ factors of $\mathbb{P}^{\mathbf{n}}$ and put $\delta_{i}:=\operatorname{dim} p_{i}(Z)-$ $\operatorname{dim} p_{i+1}(Z)$ for $i=1, \ldots, m$, where $\operatorname{dim} p_{m+1}(Z):=0$. Let $s:=\operatorname{codim} Z$. Then

$$
m_{Z}=l_{\mathcal{O}_{Z}}\left(\mathcal{O}_{Z} / I \mathcal{O}_{Z}\right) \geq(\varepsilon / s)^{s} d_{1}^{n_{1}-\delta_{1}} \ldots d_{m}^{n_{m}-\delta_{m}} .
$$

Proof. We follow the arguments of van der Put [11] and Wüstholz [20]. For convenience of the reader, we have worked out more details.

Choose $P \in Z$ such that $Z$ is smooth in $P$ (i.e. the tangent space of $Z$ at $P$ has dimension equal to that of $Z$ ) and for $i=1, \ldots, m, p_{i}(Z)$ is smooth in $p_{i}(P)$ and the map $p_{i}$ is smooth at $P$ (i.e. the linear map of tangent spaces $d p_{i}$ corresponding to $p_{i}$ is surjective). Such a point $P$ exists since by [8], Lemma 10.5, p. 271, the set of such points is a non-empty Zariski open subset of $Z$. After applying a linear transformation if need be, we may assume that $P=\left(P_{1}, \ldots, P_{m}\right)$ with $P_{h}=(1: 0: \ldots: 0) \in \mathbb{P}^{n_{h}}$ for 
$h=1, \ldots, m$. Now define the affine variety

$$
A:=\mathbb{A}^{M}=\mathbb{A}^{n_{1}} \times \ldots \times \mathbb{A}^{n_{m}}=\mathbb{P}^{\mathbf{n}} \cap\left\{X_{10} \neq 0, \ldots, X_{m 0} \neq 0\right\} .
$$

On $A$ we choose the affine coordinates $Y_{h j}=X_{h j} / X_{h 0}(h=1, \ldots, m, j=$ $\left.1, \ldots, n_{h}\right)$. Let $k[\mathbf{Y}]$ be the polynomial ring in these coordinates. Put $F^{\prime}(\mathbf{Y})$ $:=F\left(1, Y_{11}, \ldots, Y_{1, n}, \ldots, 1, Y_{m 1}, \ldots, Y_{m, n_{m}}\right)$ and let $I_{\mu}^{\prime}$ be the ideal generated by the polynomials

$$
\prod_{h=1}^{m} \prod_{j=1}^{n_{m}} \frac{\partial^{i_{h j}}}{\partial Y_{h j}^{i_{h j}}} F^{\prime} \quad \text { with } \quad \sum_{h=1}^{m} \frac{1}{d_{h}}\left(\sum_{j=1}^{n_{h}} i_{h j}\right) \leq \mu ;
$$

by the Remark at the end of Section 1, this is the defining ideal of $Z_{\mu}(f, \mathbf{d}) \cap$ $A$. Further, let $Z^{\prime}:=Z \cap A$ and $J^{\prime}=\left\{f \in k[\mathbf{Y}]: f(P)=0\right.$ for $\left.P \in Z^{\prime}\right\}$. Then $J^{\prime}$ is a minimal prime ideal containing $I_{\sigma}^{\prime}$ and also a minimal prime ideal containing $I_{\sigma+\varepsilon}^{\prime}$. The local ring of $Z^{\prime}$,

$$
\widehat{R}:=\left\{f / g: f, g \in k[\mathbf{Y}], g \notin J^{\prime}\right\}
$$

is isomorphic to $\mathcal{O}_{Z}$ and has maximal ideal $\widehat{M}:=J^{\prime} \widehat{R}$. Put $\widehat{I}:=I^{\prime} \widehat{R}$. Then $\widehat{R} / \widehat{I} \cong \mathcal{O}_{Z} / I \mathcal{O}_{Z}$. Therefore, $m_{Z}=l_{\widehat{R}}(\widehat{R} / \widehat{I})$, so it suffices to show that

$$
l_{\widehat{R}}(\widehat{R} / \widehat{I}) \geq(\varepsilon / s)^{s} d_{1}^{n_{1}-d_{1}} \ldots d_{m}^{n_{m}-\delta_{m}} .
$$

Since $\widehat{M}=\left(f_{1}, \ldots, f_{u}\right) \widehat{R}$, the tangent space of $Z^{\prime}$ at $\mathbf{0}$ is given by

$$
\begin{aligned}
T_{\mathbf{0}}\left(Z^{\prime}\right)=\left\{\mathbf{w}=\left(w_{h j}: h=\right.\right. & \left.1, \ldots, m, j=1, \ldots, n_{h}\right) \in k^{M}: \\
& \left.\sum_{h=1}^{m} \sum_{j=1}^{n_{h}} \frac{\partial f_{l}}{\partial Y_{h j}}(\mathbf{0}) w_{h j}=0 \text { for } l=1, \ldots, u\right\} .
\end{aligned}
$$

The linear mapping $d p_{i}$ induced by $p_{i}$ from $T_{\mathbf{0}}$ to the tangent space $T_{p_{i}(\mathbf{0})}\left(p_{i}\left(Z^{\prime}\right)\right)$ of $p_{i}\left(Z^{\prime}\right)$ at $p_{i}(\mathbf{0})$ can be given by $d p_{i}(\mathbf{w})=\left(w_{h j}: h=\right.$ $\left.i, \ldots, m, j=1, \ldots, n_{h}\right)$. Our smoothness assumptions at the beginning of the proof imply that $\operatorname{dim} T_{\mathbf{0}}\left(Z^{\prime}\right)=\operatorname{dim} Z^{\prime}, \operatorname{dim} T_{p_{i}(\mathbf{0})}\left(p_{i}\left(Z^{\prime}\right)\right)=\operatorname{dim} p_{i}\left(Z^{\prime}\right)=$ $\delta_{i}+\ldots+\delta_{m}$, and that $d p_{i}$ is surjective. Therefore,

$$
\left\{\begin{aligned}
\operatorname{dim} \operatorname{ker}\left(d p_{i}\right) & =\operatorname{dim} T_{\mathbf{0}}\left(Z^{\prime}\right)-\operatorname{dim} T_{p_{i}(\mathbf{0})}\left(p_{i}\left(Z^{\prime}\right)\right) \\
& =\delta_{1}+\ldots+\delta_{i-1} \quad \text { for } i=2, \ldots, m, \\
\operatorname{ker} d p_{1} & =(\mathbf{0}) .
\end{aligned}\right.
$$

Note that

$$
\begin{array}{r}
\operatorname{ker}\left(d p_{i}\right)=\left\{\mathbf{w} \in k^{M}: \sum_{h=1}^{i-1} \sum_{j=1}^{n_{h}} \frac{\partial f_{l}}{\partial Y_{h j}}(\mathbf{0}) w_{h j}=0 \text { for } l=1, \ldots, u,\right. \\
\left.w_{h j}=0 \text { for } h=i, \ldots, m, j=1, \ldots, n_{h}\right\} .
\end{array}
$$


By (4.5) and (4.6), the $\left(n_{1}+\ldots+n_{i-1}\right) \times u$-matrix

$$
A_{i}=\left(\frac{\partial f_{l}}{\partial Y_{h j}}(\mathbf{0})\right)_{\substack{h=1, \ldots, i-1, j=1, \ldots, n_{h} \\ l=1, \ldots, u}}
$$

with rows being indexed by $(h, j)$ and columns by $l$, has rank $\left(n_{1}-\delta_{1}\right)+$ $\ldots+\left(n_{i-1}-\delta_{i-1}\right)$. Hence among the rows $\left(\partial f_{l} / \partial Y_{i j}\right)(\mathbf{0})\left(j=1, \ldots, n_{i}\right)$ of $A_{i+1}$ there are precisely $n_{i}-\delta_{i}$ rows which are linearly independent of each other and also linearly independent of the rows of $A_{i}$; we assume without loss of generality that these rows are $\left(\partial f_{l} / \partial Y_{i j}\right)(\mathbf{0})$ with $j=1, \ldots, n_{i}-\delta_{i}$ and $l=1, \ldots, u$. This gives altogether $\left(n_{1}-\delta_{1}\right)+\ldots+\left(n_{m}-\delta_{m}\right)=s$ linearly independent rows $\left(\partial f_{l} / \partial Y_{h j}\right)(\mathbf{0})\left(h=1, \ldots, m, j=1, \ldots, n_{h}-\delta_{h}\right)$.

For convenience, write $Y_{1}, \ldots, Y_{s}$ for the variables $Y_{h j}(h=1, \ldots, m$, $j=1, \ldots, n_{h}-\delta_{h}$ ) and put $c_{i}=d_{h}$ whenever $Y_{i}=Y_{h j}$. Obviously (4.4) follows once we have shown that

$$
l_{\widehat{R}}(\widehat{R} / \widehat{I}) \geq(\varepsilon / s)^{s} c_{1} \ldots c_{s} .
$$

By what we have seen above, the matrix $\left(\left(\partial f_{l} / \partial Y_{j}\right)(\mathbf{0})\right)_{j=1, \ldots, s, l=1, \ldots, u}$ has rank $s$. We assume without loss of generality that $\operatorname{det}\left(\left(\partial f_{l} / \partial Y_{j}\right)(\mathbf{0})\right)_{i \leq j, l \leq s}$ is non-zero. Then

$$
D(\mathbf{Y}):=\operatorname{det}\left(\frac{\partial f_{l}}{\partial Y_{j}}\right)_{i \leq j, l \leq s} \notin J^{\prime} .
$$

Hence the elements of the inverse matrix $\left(g_{k l}\right)=\left(\partial f_{l} / \partial Y_{j}\right)^{-1}$ belong to $\widehat{R}$. Define the rational functions

$$
T_{j}:=\sum_{l=1}^{s} g_{l j} f_{l} \quad(j=1, \ldots, s) .
$$

Further, define differential operators $\partial / \partial T_{i}$ by

$$
\left(\frac{\partial}{\partial T_{1}}, \ldots, \frac{\partial}{\partial T_{s}}\right)=\left(\frac{\partial}{\partial Y_{1}}, \ldots, \frac{\partial}{\partial Y_{s}}\right)\left(\frac{\partial T_{i}}{\partial Y_{j}}\right)^{-1}
$$

where $\left(\partial T_{i} / \partial Y_{j}\right)^{-1}$ is the inverse matrix of $\left(\partial T_{i} / \partial Y_{j}\right)_{1 \leq i, j \leq s}$. Then $T_{1}, \ldots, T_{s}$ belong to $\widehat{M}$ since $g_{l j} \in \widehat{R}$ and $f_{l} \in \widehat{M}$. If $h \in \widehat{R}$ then $\partial h / \partial Y_{j} \in \widehat{R}$ for $j=1, \ldots, s$, namely if $h=f / g$ with $f, g \in k[\mathbf{Y}], g \notin J^{\prime}$, then

$$
\frac{\partial h}{\partial Y_{j}}=g^{-2}\left\{g \frac{\partial f}{\partial Y_{j}}-f \frac{\partial g}{\partial Y_{j}}\right\} \in \widehat{R} \text {. }
$$

Further,

$$
\frac{\partial T_{i}}{\partial Y_{j}}=\sum_{l=1}^{s} g_{l i} \frac{\partial f_{l}}{\partial Y_{j}}+\sum_{l=1}^{s}\left(\frac{\partial g_{l i}}{\partial Y_{j}}\right) f_{l} \equiv \delta_{i j} \bmod \widehat{M}
$$


Hence $\operatorname{det}\left(\partial T_{i} / \partial Y_{j}\right)$ is a unit in $\widehat{R}$, which implies that the elements of $\left(\partial T_{i} / \partial Y_{j}\right)^{-1}$ belong to $\widehat{R}$. It follows that $\partial h / \partial T_{j} \in \widehat{R}$ for $h \in \widehat{R}, j=$ $1, \ldots, s$. The operators $\partial / \partial T_{i}$ satisfy the usual rules for differentiation, e.g., $\partial T_{i} / \partial T_{j}=\delta_{i j}$ and $\partial T_{i}^{l} / \partial T_{i}=l T_{i}^{l-1}$ for $l=1,2, \ldots$ We have the following crucial fact:

$$
\partial^{j_{1}+\ldots+j_{s}} f / \partial T_{1}^{j_{1}} \ldots \partial T_{s}^{j_{s}} \in \widehat{M} \text { for every } f \in \widehat{I} \text { and all tuples of }
$$
non-negative integers $\left(j_{1}, \ldots, j_{s}\right)$ with $j_{1} / c_{1}+\ldots+j_{s} / c_{s} \leq \varepsilon$.

Namely, let $f \in \widehat{I}$ and $j_{1} / c_{1}+\ldots+j_{s} / c_{s} \leq \varepsilon$. $f$ can be expressed as $g_{1} f_{1}+\ldots+$ $g_{r} f_{r}$ with $g_{1}, \ldots, g_{r} \in \widehat{R}, f_{1}, \ldots, f_{r} \in I_{\sigma}^{\prime}$. Hence $\partial^{j_{1}+\ldots+j_{s}} f / \partial T_{1}^{j_{1}} \ldots \partial T_{s}^{j_{s}}$ can be expressed as $\sum h_{i k} D_{i} f_{k}$ with $h_{i k} \in \widehat{R}$ and $D_{i}=\partial^{i_{1}+\ldots+i_{s}} / \partial T_{1}^{i_{1}} \ldots \partial T_{s}^{i_{s}}$ for certain $i_{1} \leq j_{1}, \ldots, i_{s} \leq j_{s}$. Furthermore, $D_{i} f_{k}$ can be expressed as $\sum p_{l k i} D_{l}^{\prime} f_{k}$ with $p_{l k i} \in \widehat{R}$ and $D_{l}^{\prime}=\partial^{l_{1}+\ldots+l_{s}} / \partial Y_{1}^{l_{1}} \ldots \partial Y_{s}^{l_{s}}$ with $l_{1} \leq i_{1} \leq$ $j_{1}, \ldots, l_{s} \leq i_{s} \leq j_{s}$. Since $I_{\sigma+\varepsilon}^{\prime} \subseteq J^{\prime}$ we have $D_{l}^{\prime} f_{k} \in J^{\prime}$; this implies (4.8).

We are now ready to prove Lemma 10 . Define an ordering on $\left(\mathbb{Z}_{\geq 0}\right)^{s}$ by defining $\mathbf{i}<\mathbf{j}$ if the first non-zero coordinate of $\mathbf{j}-\mathbf{i}$ is $>0$. For $\mathbf{i}=\left(i_{1}, \ldots, i_{s}\right)$, put $D^{\mathbf{i}}=\partial^{i_{1}+\ldots+i_{s}} / \partial T_{1}^{i_{1}} \ldots \partial T_{s}^{i_{s}}$ and $\mathbf{T}^{\mathbf{i}}=T_{1}^{i_{1}} \ldots T_{s}^{i_{s}}$. Let $\mathbf{i}_{1}, \ldots, \mathbf{i}_{l}$ be the tuples with $i_{1} / c_{1}+\ldots+i_{s} / c_{s} \leq \varepsilon$, ordered so that $\mathbf{i}_{1}>\mathbf{i}_{2}>\ldots>\mathbf{i}_{l}$. Define the ideals in $\widehat{R}$ :

$$
\begin{aligned}
& J_{0}=\widehat{I}+\left(\mathbf{T}^{\mathbf{j}}: \text { all } \mathbf{j}=\left(j_{1}, \ldots, j_{s}\right) \text { with } j_{1} / c_{1}+\ldots+j_{s} / c_{s}>\varepsilon\right), \\
& J_{t}=J_{0}+\left(\mathbf{T}^{\mathbf{i}_{1}}, \ldots, \mathbf{T}^{\mathbf{i}_{t}}\right) \quad \text { for } t=1, \ldots, l .
\end{aligned}
$$

We have

$$
J_{0} \varsubsetneqq J_{1} \varsubsetneqq \cdots \varsubsetneqq J_{l} .
$$

Namely, suppose that for some $t$ we have $J_{t+1}=J_{t}$. Then $\mathbf{T}^{\mathbf{i}_{t+1}} \in J_{t}$, i.e. $\mathbf{T}^{\mathbf{i}_{t+1}}=\sum_{\mathbf{i}} g_{\mathbf{i}} T^{\mathbf{i}}+f$, where the sum is taken over tuples $\mathbf{i}>\mathbf{i}_{t+1}$ and where $g_{\mathbf{i}} \in \widehat{R}$ and $f \in \widehat{I}$. Since $T_{1}, \ldots, T_{s} \in \widehat{M}$ we have $D^{\mathbf{k}} \mathbf{T}^{\mathbf{i}} \in \widehat{M}$ if $\mathbf{k} \neq \mathbf{i}$. Note that $D^{\mathbf{i}_{t+1}} \mathbf{T}^{\mathbf{i}_{t+1}}$ is a non-zero constant, whence it does not belong to $\widehat{M}$. On the other hand, $D^{\mathbf{i}_{t+1}}\left(\mathbf{g}_{i} \mathbf{T}^{\mathbf{i}}\right)$ can be expressed as $\sum h_{\mathbf{i}, \mathbf{k}} D^{\mathbf{k}} \mathbf{T}^{\mathbf{i}}$ with $h_{\mathbf{i}, \mathbf{k}} \in \widehat{R}$ and $\mathbf{k} \leq \mathbf{i}_{t+1}<\mathbf{i}$, hence $D^{\mathbf{i}_{t+1}}\left(g_{\mathbf{i}} \mathbf{T}^{\mathbf{i}}\right) \in \widehat{M}$. Further, by (4.8), $D^{\mathbf{i}_{t+1}} f \in \widehat{M}$. Thus we arrive at a contradiction and we must conclude that $J_{t+1} \supseteq J_{t}$. This proves (4.9). Consequently,

$$
\widehat{R} / \widehat{I} \supseteq \widehat{R} / J_{0} \supseteq \widehat{R} / J_{1} \supseteq \ldots \supseteq \widehat{R} / J_{l} \supseteq(0) .
$$

Hence $l_{\widehat{R}}(\widehat{R} / \widehat{I}) \geq l$. The tuples $\left(i_{1}, \ldots, i_{s}\right)$ with $0 \leq i_{j} \leq\left[\varepsilon c_{j} / s\right](j=$ $1, \ldots, s)$ satisfy $i_{1} / c_{1}+\ldots+i_{s} / c_{s} \leq \varepsilon$. Hence

$$
l \geq \prod_{j=1}^{s}\left(\left[\varepsilon c_{j} / s\right]+1\right) \geq(\varepsilon / s)^{s} c_{1} \ldots c_{s} .
$$

This proves (4.7) and hence Lemma 10. 
Proof of Theorem 1 . Let $s=\operatorname{codim} Z=M-\left(\delta_{1}+\ldots+\delta_{m}\right)$, where $\delta_{1}, \ldots, \delta_{m}$ are the integers from Lemma 10. Let $\left(e_{1}, \ldots, e_{m}\right)$ be a tuple of non-negative integers with $e_{1}+\ldots+e_{m}=M-s=\delta_{1}+\ldots+\delta_{m}$ and $\left(Z \cdot \mathcal{L}_{1}^{e_{1}} \ldots \mathcal{L}_{m}^{e_{m}}\right)>0$. We have

$$
\eta_{i}:=\left(\delta_{i}+\ldots+\delta_{m}\right)-\left(e_{i}+\ldots+e_{m}\right) \geq 0 \quad \text { for } i=2, \ldots, m .
$$

Namely, take generic $f_{h j} \in \Gamma\left(\mathcal{L}_{h}\right)\left(h=1, \ldots, m, j=1, \ldots, e_{h}\right)$ and put $W:=Z \cap\left\{f_{h j}=0\right.$ for $\left.h=1, \ldots, m, j=1, \ldots, e_{h}\right\}$. Then $W$ is not empty, hence $p_{i}(W)$ is not empty. Further, $p_{i}(W) \subseteq p_{i}(Z) \cap\left\{f_{h j}=0\right.$ for $\left.h=i, \ldots, m, j=1, \ldots, e_{h}\right\}$. Hence $\operatorname{dim} p_{i}(Z)=\delta_{i}+\ldots+\delta_{m} \geq e_{i}+\ldots+e_{m}$.

From (4.3), Lemma 4 and Lemma 10 it follows that

$$
\begin{aligned}
{\left[k_{1}: k_{0}\right]\left(Z \cdot \mathcal{L}_{1}^{e_{1}} \ldots \mathcal{L}_{m}^{e_{m}}\right) } & \leq m_{Z}^{-1}\left(\mathcal{L}_{1}^{e_{1}} \ldots \mathcal{L}_{m}^{e_{m}} \cdot \mathcal{L}^{s}\right) \\
& =m_{Z}^{-1}\left(\mathcal{L}_{1}^{e_{1}} \ldots \mathcal{L}_{m}^{e_{m}} \cdot\left(d_{1} \mathcal{L}_{1}+\ldots+d_{m} \mathcal{L}_{m}\right)^{s}\right) \\
& =m_{Z}^{-1} \frac{s !}{\left(n_{1}-e_{1}\right) ! \ldots\left(n_{m}-e_{m}\right) !} d_{1}^{n_{1}-e_{1}} \ldots d_{m}^{n_{m}-e_{m}} \\
& \leq m_{Z}^{-1} m^{s} d_{1}^{n_{1}-e_{1}} \ldots d_{m}^{n_{m}-e_{m}} \\
& \leq\left(\frac{m s}{\varepsilon}\right)^{s} d_{1}^{\delta_{1}-n_{1}} \ldots d_{m}^{\delta_{m}-n_{m}} \cdot d_{1}^{n_{1}-e_{1}} \ldots d_{m}^{n_{m}-e_{m}} \\
& =\left(\frac{m s}{\varepsilon}\right)^{s}\left(\frac{d_{2}}{d_{1}}\right)^{\eta_{2}} \ldots\left(\frac{d_{m}}{d_{m-1}}\right)^{\eta_{m}}
\end{aligned}
$$

Suppose that $Z$ is not a product variety $Z_{1} \times \ldots \times Z_{m}$ with $Z_{h}$ a subvariety of $\mathbb{P}^{n_{h}}$ for $h=1, \ldots, m$. Then by Lemma 3 there are at least two tuples $\left(e_{1}, \ldots, e_{m}\right)$ with $\left(Z \cdot \mathcal{L}_{1}^{e_{1}} \ldots \mathcal{L}_{m}^{e_{m}}\right)>0$ so there is such a tuple with $\left(e_{1}, \ldots, e_{m}\right) \neq\left(\delta_{1}, \ldots, \delta_{m}\right)$. But then, at least one of the numbers $\eta_{i}$ is $\geq 1$. Together with (4.11) and condition (1.1) on $d_{1} / d_{2}, \ldots, d_{m-1} / d_{m}$ this implies that

$$
\left[k_{1}: k_{0}\right]\left(Z \cdot \mathcal{L}_{1}^{e_{1}} \ldots \mathcal{L}_{m}^{e_{m}}\right) \leq\left(\frac{m s}{\varepsilon}\right)^{s}\left(\frac{m M}{\varepsilon}\right)^{-M\left(\eta_{2}+\ldots+\eta_{m}\right)}<1,
$$

which is impossible as $\left(Z \cdot \mathcal{L}_{1}^{e_{1}} \ldots \mathcal{L}_{m}^{e_{m}}\right)$ is a positive integer. It follows that $Z$ is a product variety $Z_{1} \times \ldots \times Z_{m}$ with $Z_{h}$ a subvariety of $\mathbb{P}^{n_{h}}$ and that $e_{h}=\delta_{h}=\operatorname{dim} Z_{h}$ for $h=1, \ldots, m$. Hence $\eta_{1}=\ldots=\eta_{m}=0$. By inserting this into (4.11) and using Lemma 2(ii) we get

$$
\left[k_{1}: k_{0}\right] \operatorname{deg} Z_{1} \ldots \operatorname{deg} Z_{m}=\left[k_{1}: k_{0}\right]\left(Z \cdot \mathcal{L}_{1}^{e_{1}} \ldots \mathcal{L}_{m}^{e_{m}}\right) \leq\left(\frac{m s}{\varepsilon}\right)^{s} .
$$

This completes the proof of Theorem 1 . 
Pro of of Theorem 2 . We use the notation introduced at the beginning of this section, except that $k=\overline{\mathbb{Q}}$. We assume that $F$ has its coefficients in some number field $K$, and that the ideal generated by the coefficients of $F$ is (1). As in the proof of Lemma 7(iv), this is no restriction. The coefficients of each polynomial

$$
F_{\mathbf{i}}:=\prod_{h=1}^{m} \prod_{j=0}^{n_{h}} X_{h j}^{c_{h j}}\left(\prod_{h=1}^{m} \prod_{j=0}^{n_{h}} \frac{1}{i_{h j} !} \cdot \frac{\partial^{i_{h j}}}{\partial X_{h j}^{i_{h j}}} F\right)
$$

are obtained by multiplying the coefficient of $F$ attached to the monomial $\prod_{h=1}^{m} \prod_{j=0}^{n_{h}} X_{h j}^{l_{h j}}$ with $\prod_{h=1}^{m} \prod_{j=0}^{n_{h}}\left(\begin{array}{l}l_{h j} \\ i_{h j}\end{array}\right)$, which is an integer

$$
\leq 2^{\sum_{h=1}^{m} \Sigma_{j=1}^{n_{h}} l_{h j}} \leq 2^{d_{1}+\ldots+d_{m}} .
$$

It follows that for each embedding $\sigma: K \hookrightarrow \mathbb{C}$,

$$
H_{\sigma}\left(F_{\mathbf{i}}\right) \leq 2^{d_{1}+\ldots+d_{m}} H_{\sigma}(F)=: H_{\sigma} .
$$

Recall that the coefficients of $F$ generate the ideal (1), so that

$$
\left(\prod_{\sigma} H_{\sigma}\right)^{1 /[K: \mathbb{Q}]} \leq 2^{d_{1}+\ldots+d_{m}} H(F) .
$$

By applying Lemma 6(iv) with $f$ having coefficients in $k_{0}$ and using induction on the dimension, we see that

$$
h\left(Z^{(i)}, \mathcal{L}^{M-s+1}\right)=h\left(Z, \mathcal{L}^{M-s+1}\right) \quad \text { for } i=1, \ldots,\left[k_{1}: k_{0}\right] .
$$

Together with Lemma 9, (4.12) and (4.13) this implies

$$
\begin{aligned}
& {\left[k_{1}: k_{0}\right] m_{Z} h\left(Z, \mathcal{L}^{M-s+1}\right) } \\
\leq & \frac{M !}{n_{1} ! \ldots n_{m} !} d_{1}^{n_{1}} \ldots d_{m}^{n_{m}}\left\{\left(M^{2}+\log 2\right)\left(d_{1}+\ldots+d_{m}\right)+s \log H(F)\right\} \\
\leq & 2 m^{M} M^{2} d_{1}^{n_{1}} \ldots d_{m}^{n_{m}}\left(d_{1}+\ldots+d_{m}+\log H(F)\right) .
\end{aligned}
$$

We have shown that $Z=Z_{1} \times \ldots \times Z_{m}$, where $Z_{h}$ is a $\delta_{h}$-dimensional subvariety of $\mathbb{P}^{n_{h}}$ for $h=1, \ldots, m$. By Lemmas 10,9 and 8 (ii) we have

$$
\begin{aligned}
& {\left[k_{1}: k_{0}\right] m_{Z} h\left(Z, \mathcal{L}^{M-s+1}\right)} \\
& \geq\left[k_{1}: k_{0}\right](\varepsilon / s)^{s} d_{1}^{n_{1}-\delta_{1}} \ldots d_{m}^{n_{m}-\delta_{m}} \\
& \quad \times d_{1}^{\delta_{1}} \ldots d_{m}^{\delta_{m}} \operatorname{deg} Z_{1} \ldots \operatorname{deg} Z_{m}\left(\sum_{h=1}^{m} \frac{d_{h} h\left(Z_{h}\right)}{\operatorname{deg} Z_{h}}\right) \\
& =\left[k_{1}: k_{0}\right] \operatorname{deg} Z_{1} \ldots \operatorname{deg} Z_{m} \cdot(\varepsilon / s)^{s} d_{1}^{n_{1}} \ldots d_{m}^{n_{m}} \sum_{h=1}^{m} \frac{d_{h} h\left(Z_{h}\right)}{\operatorname{deg} Z_{h}} .
\end{aligned}
$$

By comparing this with (4.14) we see that the term $d_{1}^{n_{1}} \ldots d_{m}^{n_{m}}$ cancels and that 


$$
\begin{aligned}
{\left[k_{1}: k_{0}\right] \operatorname{deg} Z_{1} \ldots \operatorname{deg} Z_{m} } & \left(\sum_{h=1}^{m} \frac{d_{h} h\left(Z_{h}\right)}{\operatorname{deg} Z_{h}}\right) \\
\leq & 2\left(\frac{s}{\varepsilon}\right)^{s} m^{M} M^{2}\left(d_{1}+\ldots+d_{m}+\log H(F)\right),
\end{aligned}
$$

which is Theorem 2 .

5. Proof of Theorem 3 (Roth's lemma). Let $m$ be an integer $\geq 2$. Put $\mathbb{P}:=\mathbb{P}^{1}(\overline{\mathbb{Q}}) \times \ldots \times \mathbb{P}^{1}(\overline{\mathbb{Q}})(m$ times $)$ and denote by $\mathbb{P}_{i}^{1}$ the $i$ th factor of $\mathbb{P}$. Define the blocks of two variables $\mathbf{X}_{h}:=\left(X_{h 0}, X_{h 1}\right)$, for $\mathbf{d}=\left(d_{1}, \ldots, d_{m}\right) \in$ $\left(\mathbb{Z}_{\geq 0}\right)^{m}$ let $\Gamma(\mathbf{d})$ be the set of polynomials from $\overline{\mathbb{Q}}\left[\mathbf{X}_{1}, \ldots, \mathbf{X}_{m}\right]$ which are homogeneous of degree $d_{h}$ in $\mathbf{X}_{h}$ for $h=1, \ldots, m$ and let $\Gamma:=\bigcup_{\mathbf{d}} \Gamma(\mathbf{d})$. As usual, we put $\mathcal{L}_{h}=\mathcal{O}(0, \ldots, 1, \ldots, 0)$ ( 1 on the $h$ th place).

Now let $0<\varepsilon \leq m+1$ and let $\mathbf{d}=\left(d_{1}, \ldots, d_{m}\right)$ be a tuple of positive integers satisfying (1.11). Further, let $F$ be a non-zero polynomial from $\Gamma(\mathbf{d})$ and let $P=\left(P_{1}, \ldots, P_{m}\right)$, where $P_{h} \in \mathbb{P}^{1}$ for $h=1, \ldots, m$. Assume that $i_{\mathbf{d}}(F, P) \geq \varepsilon$. We shall show that for at least one $h, P_{h}$ does not satisfy (1.12), i.e.

$$
H\left(P_{h}\right)^{d_{h}} \leq\left(e^{d_{1}+\ldots+d_{m}} H(F)\right)^{\left(3 m^{3} / \varepsilon\right)^{m}} .
$$

This clearly implies Theorem 3 .

Put $\varepsilon^{\prime}:=\varepsilon /(m+1)$. As in the proof of the Corollary, there is an $i \in\{0, \ldots, m\}$ such that $Z_{i \varepsilon^{\prime}}$ and $Z_{(i+1) \varepsilon^{\prime}}$ have a common irreducible component, $Z$, say, containing $P$. Put $s:=\operatorname{codim} Z$. As in Lemma 10 , let $p_{i}$ be the projection of $\mathbb{P}$ onto the product of its last $m-i+1$ factors $\mathbb{P}_{i}^{1} \times \ldots \times \mathbb{P}_{m}^{1}$ and put $\delta_{i}:=\operatorname{dim} p_{i}(Z)-\operatorname{dim} p_{i+1}(Z)$ for $i=1, \ldots, m$, where $\operatorname{dim} p_{m+1}(Z):=0$; note that $\delta_{i} \in\{0,1\}$. Further, let $\pi_{h}$ be the projection of $\mathbb{P}$ onto its $h$ th factor $\mathbb{P}_{h}^{1}$. Then either $\pi_{h}(Z)=\mathbb{P}_{h}^{1}$ or $\pi_{h}(Z)$ is a point, in which case $\pi_{h}(Z)=P_{h}$. We shall show that for some $h$ we have $\pi_{h}(Z)=P_{h}$ and that this $P_{h}$ satisfies (5.1). To this end we need the following improvement of Lemma 3 for the case $\mathbf{n}=(1, \ldots, 1)$.

LEMma 11. There are $e_{1}, \ldots, e_{m} \in\{0,1\}$ with $e_{1}+\ldots+e_{m}=\operatorname{dim} Z=$ $m-s,\left(Z \cdot \mathcal{L}_{1}^{e_{1}} \ldots \mathcal{L}_{m}^{e_{m}}\right)>0, \eta_{i}:=\sum_{j=i}^{m}\left(\delta_{j}-e_{j}\right) \geq 0$ for $i=2, \ldots, m$ and

$$
\sum_{i=2}^{m} \eta_{i} \geq\left(\sum_{i=1}^{m} \operatorname{dim} \pi_{i}(Z)\right)-\operatorname{dim} Z .
$$

Proof. For any subset $\mathbf{i}=\left\{i_{1}, \ldots, i_{t}\right\}$ of $\{1, \ldots, m\}$ denote by $\pi_{\mathbf{i}}$ the projection of $\mathbb{P}$ onto $\mathbb{P}_{i_{1}}^{1} \times \ldots \times \mathbb{P}_{i_{t}}^{1}$ and put $c_{\mathbf{i}}:=\operatorname{dim} \pi_{\mathbf{i}}(Z)$.

We proceed by induction on $m$. For $m=1$, Lemma 11 is trivial. Suppose that $m \geq 2$. For the moment, suppose also that $\pi_{1}(Z)=\mathbb{P}_{1}^{1}$. Let $X$ be the set of points $P$ in $Z$ such that for some $\mathbf{i} \subseteq\{1, \ldots, m\}$ either $\pi_{\mathbf{i}}(Z)$ 
is not smooth at $\pi_{\mathbf{i}}(P)$ or the restriction $\pi_{\mathbf{i}} \mid Z$ of $\pi_{\mathbf{i}}$ to $Z$ is not smooth at $P$. Then $X$ is a proper, Zariski-closed subset of $Z$. For $Q=(p: q) \in \mathbb{P}_{1}^{1}$, let $f_{Q}=q X_{10}-p X_{11}, Z_{Q}=Z \cap\left\{f_{Q}=0\right\}$. There are only finitely many $Q \in \mathbb{P}_{1}^{1}$ such that one of the irreducible components of $Z_{Q}$ is contained in $X$. Namely, $X$ has only finitely many irreducible components and if some irreducible component $Z^{\prime}$ of $Z_{Q}$ is contained in $X$, then $Z^{\prime}$ is an irreducible component of $X$ since $\operatorname{dim} Z^{\prime}=\operatorname{dim} Z-1$. Now choose $Q \in \mathbb{P}_{1}^{1}$ such that no irreducible component of $Z_{Q}$ is contained in $X$ and let $Z^{\prime}$ be any irreducible component of $Z_{Q}$. We are going to apply the induction hypothesis to $Z^{\prime}$.

We have to consider tangent spaces at an appropriate point. Choose $P \in Z^{\prime}$ such that for each $\mathbf{i} \subseteq\{1, \ldots, m\}, \pi_{\mathbf{i}}(Z)$ is smooth at $\pi_{\mathbf{i}}(P), \pi_{\mathbf{i}} \mid Z$ is smooth at $P, \pi_{\mathbf{i}}\left(Z^{\prime}\right)$ is smooth at $\pi_{\mathbf{i}}(P)$ and the restriction $\pi_{\mathbf{i}} \mid Z^{\prime}$ is smooth at $P$. Such a $P$ exists since $Z^{\prime}$ is not contained in $X$ and the set of $P \in Z^{\prime}$ such that for some $\mathbf{i} \subseteq\{1, \ldots, m\}$ either $\pi_{\mathbf{i}}\left(Z^{\prime}\right)$ is not smooth at $\pi_{\mathbf{i}}(P)$ or $\pi_{\mathbf{i}} \mid Z^{\prime}$ is not smooth at $P$ is a proper Zariski-closed subset of $Z^{\prime}$.

We assume without loss of generality that $P=(1: 0 ; \ldots ; 1: 0)$. Let $\mathbb{A}:=\left\{X_{10} \neq 0, \ldots, X_{m 0} \neq 0\right\}$ and define affine coordinates $Y_{1}=$ $X_{11} / X_{10}, \ldots, Y_{m}=X_{m 1} / X_{m 0}$. Thus, $Z^{\prime} \cap \mathbb{A}$ is an irreducible component of $(Z \cap \mathbb{A}) \cap\left\{Y_{1}=0\right\}$. There are polynomials $f_{1}, \ldots, f_{r} \in \overline{\mathbb{Q}}\left[Y_{1}, \ldots, Y_{m}\right]$ such that $Z \cap \mathbb{A}=\left\{\mathbf{y} \in \mathbb{A}: f_{1}(\mathbf{y})=\ldots=f_{r}(\mathbf{y})=0\right\}$. The tangent space of $Z$ at $P$ is given by

$$
T:=\left\{\mathbf{y}=\left(y_{1}, \ldots, y_{m}\right) \in \overline{\mathbb{Q}}^{m}: \sum_{j=1}^{m}\left(\partial f_{i} / \partial Y_{j}\right)(\mathbf{0}) y_{j}=0 \text { for } i=1, \ldots, r\right\} .
$$

Since $\pi_{\mathbf{i}} \mid Z$ is smooth at $P$, the linear map $d \pi_{\mathbf{i}}$ corresponding to $\pi_{\mathbf{i}}$, which is the projection $\mathbf{y} \mapsto\left(y_{i}: i \in \mathbf{i}\right)$, maps $T$ surjectively to the tangent space $T_{\mathbf{i}}$ of $\pi_{\mathbf{i}}(Z)$ at $\pi_{\mathbf{i}}(P)$. Since $Z$ is smooth at $P$ we have $\operatorname{dim} T=\operatorname{dim} Z$ and since $\pi_{\mathbf{i}}(Z)$ is smooth at $\pi_{\mathbf{i}}(P)$ we have $\operatorname{dim} T_{\mathbf{i}}=\operatorname{dim} \pi_{\mathbf{i}}(Z)=c_{\mathbf{i}}$.

Similarly, $d \pi_{\mathbf{i}}$ maps the tangent space $T^{\prime}$ of $Z^{\prime}$ at $P$ surjectively to the tangent space $T_{\mathbf{i}}^{\prime}$ of $\pi_{\mathbf{i}}\left(Z^{\prime}\right)$ at $\pi_{\mathbf{i}}(P)$ and $\operatorname{dim} T_{\mathbf{i}}^{\prime}=\operatorname{dim} \pi_{\mathbf{i}}\left(Z^{\prime}\right)$. Since $Y_{1} \equiv 0$ on $Z^{\prime} \cap \mathbb{A}$ we have $y_{1} \equiv 0$ on $T^{\prime}$. Hence $T^{\prime} \subseteq T \cap\left\{y_{1}=0\right\}$. Further, $y_{1}$ is not identically zero on $T$ since $\operatorname{dim} f_{\{1\}}(T)=\operatorname{dim} Z_{1}=1$ and $\operatorname{dim} T^{\prime}=$ $\operatorname{dim} Z^{\prime}=\operatorname{dim} Z-1=\operatorname{dim} T-1$. Hence $T^{\prime}=T \cap\left\{y_{1}=0\right\}$.

We consider $y_{1}, \ldots, y_{m}$ as linear functions on $T$. Thus, for $\mathbf{i} \subseteq\{1, \ldots, m\}$ we have $c_{\mathbf{i}}=\operatorname{dim} T_{\mathbf{i}}=\operatorname{rank}\left\{y_{i}: i \in \mathbf{i}\right\}$.

We have the following crucial fact:

$$
\operatorname{dim} \pi_{\mathbf{i}}\left(Z^{\prime}\right)=\left\{\begin{array}{l}
\operatorname{dim} \pi_{\mathbf{i}}(Z) \\
\text { for each subset } \mathbf{i} \text { of }\{1, \ldots, m\} \text { with } c_{\{1\} \cup \mathbf{i}}>c_{\mathbf{i}}, \\
\operatorname{dim} \pi_{\mathbf{i}}(Z)-1 \\
\text { for each subset } \mathbf{i} \text { of }\{1, \ldots, m\} \text { with } c_{\{1\} \cup \mathbf{i}}=c_{\mathbf{i}} .
\end{array}\right.
$$


Namely, for $\mathbf{i} \subseteq\{1, \ldots, m\}$ let $V_{\mathbf{i}}=\operatorname{ker} d \pi_{\mathbf{i}} \cap T=T \cap\left\{y_{i}=0\right.$ for $\left.i \in \mathbf{i}\right\}$, $V_{\mathbf{i}}^{\prime}=\operatorname{ker} d \pi_{\mathbf{i}} \cap T^{\prime}=T^{\prime} \cap\left\{y_{i}=0\right.$ for $\left.i \in \mathbf{i}\right\}$. Thus, $V_{\mathbf{i}}^{\prime}=V_{\{1\} \cup \mathbf{i}}$. Further, put $e_{\mathbf{i}}=c_{\{1\} \cup \mathbf{i}}-c_{\mathbf{i}}$; then $e_{\mathbf{i}} \in\{0,1\}$. Now for $\mathbf{i} \subseteq\{1, \ldots, m\}$ we have

$$
\begin{aligned}
\operatorname{dim} \pi_{\mathbf{i}}\left(Z^{\prime}\right) & =\operatorname{dim} T^{\prime}-\operatorname{dim} V_{\mathbf{i}}^{\prime}=\operatorname{dim} T-1-\operatorname{dim} V_{\{1\} \cup \mathbf{i}} \\
& =\operatorname{dim} \pi_{\{1\} \cup \mathbf{i}}\left(Z^{\prime}\right)-1=\operatorname{dim} \pi_{\mathbf{i}}(Z)+e_{\mathbf{i}}-1,
\end{aligned}
$$

which is precisely (5.2).

We now complete the induction step. Put $\delta_{i}^{\prime}:=\operatorname{dim} p_{i}\left(Z^{\prime}\right)-\operatorname{dim} p_{i+1}\left(Z^{\prime}\right)$ for $i=1, \ldots, m$, where $\operatorname{dim} p_{m+1}\left(Z^{\prime}\right):=0$. Put also $c_{i}:=\operatorname{dim} \pi_{i}(Z), c_{i}^{\prime}:=$ $\operatorname{dim} \pi_{i}\left(Z^{\prime}\right)$. Recall that $Z^{\prime}=Q \times W$, where $Q \in \mathbb{P}_{1}^{1}$ and $W$ is a subvariety of $\mathbb{P}_{2}^{1} \times \ldots \times \mathbb{P}_{m}^{1}$. By applying the induction hypothesis to $W$ we infer that there are $e_{2}, \ldots, e_{m} \in\{0,1\}$ such that

$$
\begin{gathered}
e_{2}+\ldots+e_{m}=\operatorname{dim} Z^{\prime}=\operatorname{dim} Z-1, \\
\left(Z^{\prime} \cdot \mathcal{L}_{2}^{e_{2}} \ldots \mathcal{L}_{m}^{e_{m}}\right)>0, \\
\eta_{i}^{\prime}:=\sum_{j=i}^{m}\left(\delta_{j}^{\prime}-e_{j}\right) \geq 0 \quad \text { for } i=3, \ldots, m, \\
\eta_{3}^{\prime}+\ldots+\eta_{m}^{\prime} \geq \sum_{j=2}^{m} c_{j}^{\prime}-\operatorname{dim} Z^{\prime} .
\end{gathered}
$$

Put $e_{1}=1$. Obviously, $\left(Z \cdot \mathcal{L}_{1}^{e_{1}} \ldots \mathcal{L}_{m}^{e_{m}}\right)>0$. Let $t$ be the largest index such that $y_{1}$ is linearly dependent on $\left\{y_{i}: i \geq t\right\}$. Then $\delta_{t}=\operatorname{dim} p_{t}(Z)-$ $\operatorname{dim} p_{t+1}(Z)=\operatorname{rank}\left\{y_{i}: i \geq t\right\}-\operatorname{rank}\left\{y_{i}: i \geq t+1\right\}=1$. Further, by (5.2) we have $\delta_{i}^{\prime}=\delta_{i}, \eta_{i}^{\prime}=\eta_{i}, c_{i}^{\prime}=c_{i}$ for $i>t, \delta_{t}^{\prime}=\delta_{t}-1=0, \delta_{i}^{\prime}=\delta_{i}$ for $2 \leq i<t$ and $\eta_{i}^{\prime}=\eta_{i}-1$ for $2 \leq i \leq t$, where $\eta_{2}^{\prime}:=\sum_{j=2}^{m}\left(\delta_{j}^{\prime}-e_{j}\right)=0$. Moreover, $c_{i}^{\prime} \geq c_{i}-1$ for $i \leq t$ and $\operatorname{dim} Z^{\prime}=\operatorname{dim} Z-1$. It follows that

$$
\begin{aligned}
\sum_{j=2}^{m} \eta_{j} & =\sum_{j=3}^{m} \eta_{j}^{\prime}+t-1 \\
& \geq\left(\sum_{j=2}^{m} c_{j}^{\prime}\right)+t-1-\operatorname{dim} Z^{\prime} \geq\left(\sum_{j=1}^{m} c_{j}\right)-\operatorname{dim} Z .
\end{aligned}
$$

This completes the induction step for the case $\operatorname{dim} \pi_{1}(Z)>0$. In the other case we have $Z=Q \times W$, where $Q \in \mathbb{P}_{1}^{1}$ and $W$ is a subvariety of $\mathbb{P}_{2}^{1} \times \ldots \times$ $\mathbb{P}_{m}^{1}$, and then the induction step is completed by applying the induction hypothesis to $W$. This proves Lemma 11 .

Proof of Theorem 3 . Suppose that the integers $d_{1}, \ldots, d_{m}$ satisfy (1.11), i.e. $d_{h} / d_{h+1}>2 m^{3} / \varepsilon$ for $h=1, \ldots, m-1$. Put $\mathcal{L}:=d_{1} \mathcal{L}_{1}+\ldots+$ $d_{m} \mathcal{L}_{m}$. Let $e_{1}, \ldots, e_{m}$ be the integers from Lemma 11. Assume that $\pi_{h}(Z)=$ 
$\mathbb{P}_{h}^{1}$ for $h=1, \ldots, m$. Then by Lemma 11,

$$
\eta_{2}+\ldots+\eta_{m} \geq \operatorname{codim} Z=s .
$$

Together with (4.11) (cf. proof of Theorem 1 with $i \varepsilon^{\prime}, \varepsilon^{\prime}$ replacing $\sigma, \varepsilon$, respectively) and $\eta_{1}=0$ this implies that

$$
\begin{aligned}
1 & \leq\left(Z \cdot \mathcal{L}_{1}^{e_{1}} \ldots \mathcal{L}_{m}^{e_{m}}\right) \leq\left(\frac{m s}{\varepsilon^{\prime}}\right)^{s}\left(\frac{d_{2}}{d_{1}}\right)^{\eta_{2}} \ldots\left(\frac{d_{m}}{d_{m-1}}\right)^{\eta_{m}} \\
& <\left(\frac{m(m+1)}{\varepsilon}\right)^{s}\left(\frac{2 m^{3}}{\varepsilon}\right)^{-s} \leq 1,
\end{aligned}
$$

which is impossible. Therefore there is an $h \in\{1, \ldots, m\}$ with $\pi_{h}(Z)=P_{h}$.

We now show that $P_{h}$ satisfies (5.1). By precisely the same argument as in the proof of Theorem 2 we have

$$
\begin{aligned}
h(Z, & \left.\mathcal{L}^{m-s+1}\right) \\
& \leq m_{Z}^{-1} \cdot m ! d_{1} \ldots d_{m}\left(\left(m^{2}+\log 2\right)\left(d_{1}+\ldots+d_{m}\right)+s \log H(F)\right) \\
& \leq\left(\frac{s}{\varepsilon^{\prime}}\right)^{s} 2 m^{2} m ! \cdot d_{1}^{\delta_{1}} \ldots d_{m}^{\delta_{m}}\left(d_{1}+\ldots+d_{m}+\log H(F)\right) \\
& \leq\left(\frac{3 m^{3}}{\varepsilon}\right)^{m} d_{1}^{\delta_{1}} \ldots d_{m}^{\delta_{m}}\left(d_{1}+\ldots+d_{m}+\log H(F)\right) .
\end{aligned}
$$

By Lemmas 6 and 7 we have

$$
\begin{aligned}
h\left(Z, \mathcal{L}^{m-s+1}\right) & =\sum_{f_{1}+\ldots+f_{m}=m-s+1} \frac{(m-s+1) !}{f_{1} ! \ldots f_{m} !} d_{1}^{f_{1}} \ldots d_{m}^{f_{m}} h\left(Z, \mathcal{L}_{1}^{f_{1}} \ldots \mathcal{L}_{m}^{f_{m}}\right) \\
& \geq d_{1}^{e_{1}} \ldots d_{m}^{e_{m}} \cdot d_{h} \cdot h\left(Z, \mathcal{L}_{1}^{e_{1}} \ldots \mathcal{L}_{h}^{e_{h}+1} \ldots \mathcal{L}_{m}^{e_{m}}\right) .
\end{aligned}
$$

Together with (5.3) and $\eta_{i} \geq 0$ for $i=2, \ldots, m$ this implies

$$
\begin{array}{rl}
d_{h} & h\left(Z, \mathcal{L}_{1}^{e_{1}} \ldots \mathcal{L}_{h}^{e_{h}+1} \ldots \mathcal{L}_{m}^{e_{m}}\right) \\
& \leq\left(\frac{3 m^{3}}{\varepsilon}\right)^{m} d_{1}^{\delta_{1}-e_{1}} \ldots d_{m}^{\delta_{m}-e_{m}}\left(d_{1}+\ldots+d_{m}+\log H(F)\right) \\
& \leq\left(\frac{3 m^{3}}{\varepsilon}\right)^{m}\left(\frac{d_{2}}{d_{1}}\right)^{\eta_{2}} \ldots\left(\frac{d_{m}}{d_{m-1}}\right)^{\eta_{m}}\left(d_{1}+\ldots+d_{m}+\log H(F)\right) \\
& \leq\left(\frac{3 m^{3}}{\varepsilon}\right)^{m}\left(d_{1}+\ldots+d_{m}+\log H(F)\right) .
\end{array}
$$

It is no restriction to assume that $P_{h}=(a: b)$ where $a, b$ belong to some number field $K$ and $(a, b)=(1)$. Then there are $\alpha, \beta \in O_{K}$ with $\alpha a+\beta b=1$. Put $f=\alpha X_{h 0}+\beta X_{h 1}$. Then $\operatorname{div}(f \mid Z)=0$ and $\kappa_{\wp}=\kappa_{\wp}\left(Z, f, \mathcal{L}_{1}^{e_{1}} \ldots \mathcal{L}_{m}^{e_{m}}\right)=$ 0 for each non-zero prime ideal $\wp$ of $O_{K}$. Further, for each embedding $\sigma$ : $K \hookrightarrow \mathbb{C}$ we see that $\|\sigma(f)\|=\left(|\sigma(a)|^{2}+|\sigma(b)|^{2}\right)^{-1 / 2}$ is constant on $Z \times{ }_{\sigma} \mathbb{C}$. 
Hence, using (3.1),

$$
\begin{aligned}
\kappa_{\sigma} & =\kappa_{\sigma}\left(Z, f, \mathcal{L}_{1}^{e_{1}} \ldots \mathcal{L}_{m}^{e_{m}}\right) \\
& =-\frac{1}{[K: \mathbb{Q}]} \int_{Z \times_{\sigma} \mathbb{C}} \log \|\sigma(f)\| \cdot c_{1}\left(\mathcal{L}_{1}\right)^{e_{1}} \wedge \ldots \wedge c_{1}\left(\mathcal{L}_{m}\right)^{e_{m}} \\
& =-\frac{1}{[K: \mathbb{Q}]} \log \left(\left(|\sigma(a)|^{2}+|\sigma(b)|^{2}\right)^{-1 / 2}\right) \int_{Z \times_{\sigma} \mathbb{C}} c_{1}\left(\mathcal{L}_{1}\right)^{e_{1}} \wedge \ldots \wedge c_{1}\left(\mathcal{L}_{m}\right)^{e_{m}} \\
& =\log \left(\left(|\sigma(a)|^{2}+|\sigma(b)|^{2}\right)^{1 / 2[K: \mathbb{Q}]}\right) \cdot\left(Z \cdot \mathcal{L}_{1}^{e_{1}} \ldots \mathcal{L}_{m}^{e_{m}}\right) .
\end{aligned}
$$

By inserting this into (3.5) and using that $(a, b)=(1)$ we obtain

$$
\begin{aligned}
h\left(Z, \mathcal{L}_{1}^{e_{1}} \ldots \mathcal{L}_{h}^{e_{h}+1} \ldots \mathcal{L}_{m}^{e_{m}}\right) & =\sum_{\sigma} \kappa_{\sigma}+\sum_{\wp} \kappa_{\wp} \\
& =\log H\left(P_{h}\right) \cdot\left(Z \cdot \mathcal{L}_{1}^{e_{1}} \ldots \mathcal{L}_{m}^{e_{m}}\right) \geq \log H\left(P_{h}\right) .
\end{aligned}
$$

Together with (5.4) this implies (5.1). This completes the proof of Theorem 3.

\section{References}

[1] G. Faltings, Diophantine approximation on abelian varieties, Ann. of Math. 133 (1991), 549-576.

[2] G. Faltings and G. Wüstholz, Diophantine approximations on projective spaces, Invent. Math. 116 (1994), 109-138.

[3] R. Ferretti, An effective version of Faltings' Product Theorem, Forum Math., to appear.

[4] W. Fulton, Intersection Theory, Band 2, Ergeb. Math. Grenzgeb. (3), Springer, Berlin, 1984.

[5] H. Gillet and C. Soulé, Arithmetic intersection theory, Inst. Hautes Études Sci. Publ. Math. 72 (1990), 93-174.

[6] P. Griffiths and J. Harris, Principles of Algebraic Geometry, Wiley, New York, 1978.

[7] W. Gubler, Höhentheorie, Math. Ann. 298 (1994), 427-456.

[8] R. Hartshorne, Algebraic Geometry, Springer, Berlin, 1977.

[9] J. de Jong, Ample line bundles and intersection theory, in: Diophantine Approximation and Abelian Varieties, Proc. conf. Soesterberg, Netherlands, 1992, B. Edixhoven and J.-H. Evertse (eds.), Lecture Notes in Math. 1566, Springer, Berlin, 1993, 69-76.

[10] P. Philippon, Sur des hauteurs alternatives, I, Math. Ann. 289 (1991), 255-283.

[11] M. van der Put, The Product theorem, in: Diophantine Approximation and Abelian Varieties, Proc. conf. Soesterberg, Netherlands, 1992, B. Edixhoven and J.-H. Evertse (eds.), Lecture Notes in Math. 1566, Springer, Berlin, 1993, 77-82.

[12] K. F. Roth, Rational approximations to algebraic numbers, Mathematika 2 (1955), 1-20; Corrigendum, ibid., 168.

[13] H. P. Schlickewei, An explicit upper bound for the number of solutions of the S-unit equation, J. Reine Angew. Math. 406 (1990), 109-120. 
[14] H. P. Schlickewei, The quantitative Subspace Theorem for number fields, Compositio Math. 82 (1992), 245-273.

[15] W. M. Schmidt, Norm form equations, Ann. of Math. 96 (1972), 526-551.

[16] - , The subspace theorem in diophantine approximations, Compositio Math. 69 (1989), 121-173.

[17] —, The number of solutions of norm form equations, Trans. Amer. Math. Soc. 317 (1990), 197-227.

[18] I. R. Shafarevich, Basic Algebraic Geometry, Springer, Berlin, 1977.

[19] C. Soulé, Géométrie d'Arakelov et théorie des nombres transcendants, in: Journées Arithmétiques de Luminy, 1989, G. Lachaud (ed.), Astérisque 198-199-200 (1991), 355-372.

[20] G. Wüstholz, Multiplicity estimates on group varieties, Ann. of Math. 129 (1989), 471-500.

[21] O. Zariski and P. Samuel, Commutative Algebra, Vol. II, Springer, Berlin, 1960.

DEPARTMENT OF MATHEMATICS AND COMPUTER SCIENCE

UNIVERSITY OF LEIDEN

P.O. BOX 9512

2300 RA LEIDEN, THE NETHERLANDS

E-mail: EVERTSE@WI.LEIDENUNIV.NL

Received on 15.7.1994

and in revised form on 16.3.1995 